\title{
Interaction of supersymmetric nonlinear sigma models with external higher spin superfields via higher spin supercurrents
}

\author{
I.L. Buchbinder, ${ }^{a, b, c}$ S. James Gates Jr. ${ }^{d}$ and Konstantinos Koutrolikos ${ }^{e}$ \\ ${ }^{a}$ Department of Theoretical Physics, Tomsk State Pedagogical University, \\ Kievskaya, Tomsk 634041, Russia \\ ${ }^{b}$ National Research Tomsk State University, \\ Lenina avenue, Tomsk 634050, Russia \\ ${ }^{c}$ Departamento de Física, ICE, Universidade Federal de Juiz de Fora, \\ Campus Universitário-Juiz de Fora, 36036-900, MG, Brazil \\ ${ }^{d}$ Department of Physics, Brown University, \\ Box 1843, 182 Hope Street, Barus 6 Holley 545, Providence, RI 02912, U.S.A. \\ e Institute for Theoretical Physics and Astrophysics, Masaryk University, \\ Kotlarska, 61137 Brno, Czech Republic \\ E-mail: joseph@tspu.edu.ru, sylvester_gates@brown.edu, \\ kkoutrolikos@physics.muni.cz
}

ABSTRACT: We consider a four dimensional generalized Wess-Zumino model formulated in terms of an arbitrary Kähler potential $\mathcal{K}(\Phi, \bar{\Phi})$ and an arbitrary chiral superpotential $\mathcal{W}(\Phi)$. A general analysis is given to describe the possible interactions of this theory with external higher spin gauge superfields of the $(s+1, s+1 / 2)$ supermultiplet via higher spin supercurrents. It is shown that such interactions do not exist beyond supergravity $(s \geq 2)$ for any $\mathcal{K}$ and $\mathcal{W}$. However, we find three exceptions, the theory of a free massless chiral, the theory of a free massive chiral and the theory of a free chiral with linear superpotential. For the first two, the higher spin supercurrents are known and for the third one we provide the explicit expressions. We also discuss the lower spin supercurrents. As expected, a coupling to (non-minimal) supergravity $(s=1)$ can always be found and we give the generating supercurrent and supertrace for arbitrary $\mathcal{K}$ and $\mathcal{W}$. On the other hand, coupling to the vector supermultiplet $(s=0)$ is possible only if $\mathcal{K}=\mathcal{K}(\bar{\Phi} \Phi)$ and $\mathcal{W}=0$.

KEYWORDS: Superspaces, Supersymmetric Effective Theories

ARXIV EPRINT: 1804.08539 


\section{Contents}

1 Introduction 1

2 Noether's method for supersymmetric nonlinear sigma model and higher spins

3 Coupling to vector supermultiplet

4 Coupling to (non-minimal) supergravity $\quad 8$

4.1 Improvement terms 8

$\begin{array}{lll}4.2 & \text { Conservation equation } & 8\end{array}$

$\begin{array}{lll}4.3 & \text { Reality condition } & 9\end{array}$

5 Coupling to higher spin supermultiplets $\quad 10$

5.1 Improvement terms 11

$\begin{array}{ll}5.2 \text { Additional freedom } & 12\end{array}$

$\begin{array}{lll}5.3 \text { Conservation equation } & 13\end{array}$

$\begin{array}{lll}5.4 \text { Reality condition } & 15\end{array}$

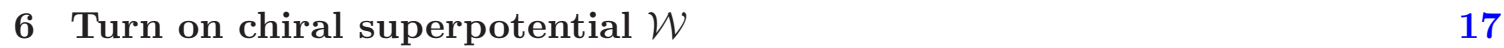

$\begin{array}{lll}6.1 & \text { For vector supermultiplet } & 18\end{array}$

$\begin{array}{lll}6.2 & \text { For supergravity } & 18\end{array}$

$\begin{array}{lll}6.3 & \text { For higher spin supermultiplet } & 19\end{array}$

$\begin{array}{lll}7 & \text { Summary and discussion } & 21\end{array}$

\section{Introduction}

Higher spin fields and their interactions are the subjects of extensive study. Despite the various no-go theorems $[1,3-5,7-18]^{1}$ and great efforts it is not clear yet whether higher spin fields play a role in the description of fundamental physical phenomena. Nevertheless, higher spin fields attract much attention due to many remarkable features, e.g. their contribution in the softness of string interactions by regularizing the ultraviolet with an infinite tower of massive states and providing a framework for studying and understanding the $A d S / C F T$ correspondence. Furthermore, studying higher spin fields allows us to better understand the structure of interactions in general gauge theory. In many cases, interaction terms for higher spins were successfully constructed for flat spacetime at first order in coupling constant $g$ by using a variety of techniques, such as light-cone approach [19-28],

\footnotetext{
${ }^{1}$ Pedagogical review in refs. $[2,6]$.
} 
Noether's procedure [10, 29-32] (some of these results were later generalized in [33-36]) and BRST [37-44]. In an intriguing manner, most of the previously mentioned results together with some new interaction vertices have been obtained by analyzing tree level amplitudes of (super)strings [45-47], thus enhancing the connection between string theory and higher spin fields. For (A)dS backgrounds similar results have been obtained [48-52] which eventually led to the fully interacting equations of motion for higher spin fields [53].

Among these interactions, the simplest class is provided by the cubic coupling of higher spin fields with low spin matter fields, such as scalar and spinor fields [31, 43, 54, 55] (and [56-60] for supersymmetric generalizations) which are of the type higher spin gauge field $\times$ conserved current, where the conserved current is quadratic in the derivatives of the matter fields. The cubic nature of these interactions is a consequence of the fact that we couple non-interacting (free) matter fields to higher spins. It is natural to take the next step and consider the coupling of interacting low spin fields to external higher spin gauge field. In this paper we ask this question and investigate the possibility of such interactions.

In order to include both scalar and spinorial matter fields in our discussion and simplify the technical details imposed by supersymmetry we will consider a theory of the chiral supermultiplet described by a chiral superfield $\Phi$. For its dynamics we will assume a nonlinear supersymmetric sigma model described by an arbitrary Kähler potential $\mathcal{K}(\Phi, \bar{\Phi})$ with the addition of an arbitrary chiral superpotential $\mathcal{W}(\Phi)$ (see e.g. [62]). Such a model is a good parametrization of many interacting matter theories and a good candidate for exploring the possible interactions with higher spins by constructing a higher spin supercurrent multiplet.

It is known that any $\mathcal{N}=1$ supersymmetric matter theory can be consistently coupled to supergravity with the help of the gravitational superfield. For that case the calculation of the conserved supercurrent is straightforward. One has to take the functional derivative of the interacting action with respect the gravitational superfield (see e.g. [62]). However, this procedure is not applicable for higher spin theory because we do not know the fully interacting theory at present. The only alternative option we have is to follow Noether's method in order to construct directly the higher spin supercurrent multiplet of the theory. However, in the case of coupling to supergravity we should be sure that the Noether procedure leads to the same supercurrent as the supergravity procedure.

In this paper, we are following a Noether-type approach and we search for the higher spin supercurrent multiplet that generates the first order coupling of the interacting matter theory with the higher spin supermultiplets of type $(s+1, s+1 / 2)$. We find that interactions with higher spin supermultiplets beyond supergravity $(s \geq 2)$ are not possible for any $\mathcal{K}$ and $\mathcal{W}$ and thus extending the results of the no-go Coleman-Mandula theorem. ${ }^{2}$ However, we find three exceptions to this rule and these are $(i)$ a free massless chiral superfield, $(i i)$ a free massive chiral superfield and (iii) a free chiral superfield with a linear superpotential. For the first two, the higher spin supercurrents and supertraces have been constructed in $[56-58,60]$. We add to this list the expressions for the supercurrent and supertrace for the third theory.

\footnotetext{
${ }^{2}$ Examples of bypassing the Coleman-Mandula theorem are discussed in [61].
} 
We also consider lower spin supercurrents. As mentioned previously, unlike the higher spin case, coupling of the theory under consideration with non-minimal supergravity can always be found for any $\mathcal{K}$ and $\mathcal{W}$. Indeed, this follows from our analysis and we get expression compatible with the results of [63]. On the other hand, interactions with the vector supermultiplet do not always exist. We find the necessary and sufficient conditions are the existence of a redefinition of the chiral superfield $\Phi \rightarrow \varphi$ such that the chiral superpotential vanishes $(\mathcal{W}=0)$ and the Kähler potential depends only in the product of $\bar{\varphi} \varphi(\mathcal{K}=\mathcal{K}(\bar{\varphi} \varphi))$. These conditions can be understood as the requirements for the presence of a global $U(1)$ symmetry which is usually associated with the vector supermultiplet.

The paper is organized as follows. In section two, we review Noether's method and its application for the construction of first order in $g$ interaction vertices. In addition, we review the description of free $4 D, \mathcal{N}=1$ higher spin supermultiplets and the conservation equation of the supercurrent multiplet. In section three, we focus on the vector supermultiplet and go through the requirements in order to construct a conserved current out of the chiral theory. In section four, we repeat the procedure for supergravity and similarly in section five for higher spin supermultiplets. In previous sections we had the chiral superpotential $\mathcal{W}$ turned of. In section six, we turn it back on and consider its contribution to the supercurrent multiplets. Finally, in section seven we review and discuss our results.

\section{Noether's method for supersymmetric nonlinear sigma model and higher spins}

The fundamental principles that govern higher spin interactions are still not understood. Hence, the only guiding principle one has, is the physical requirement of preserving the propagating degrees of freedom. This is manifested through gauge invariance. Noether's method is the framework where one organizes the invariance requirement order by order in a perturbative expansion around a starting point $S_{0}$. In this approach the full action $S[\phi, h]$ and transformation of fields $\phi, h$ are expanded in a power series of a coupling constant $g$ :

$$
\begin{aligned}
S[\phi, h] & =S_{0}[\phi]+g S_{1}[\phi, h]+g^{2} S_{2}[\phi, h]+\ldots, \\
\delta \phi & =\delta_{0}[\xi]+g \delta_{1}[\phi, \xi]+g^{2} \delta_{2}[\phi, \xi]+\ldots \\
\delta h & =\delta_{0}[\zeta]+g \delta_{1}[h, \zeta]+g^{2} \delta_{2}[h, \zeta]+\ldots
\end{aligned}
$$

The first order in $g$ interaction terms are given by $S_{1}$ and the requirement of invariance for this order gives:

$$
\frac{\delta S_{0}}{\delta \phi} \delta_{1} \phi+\frac{\delta S_{1}}{\delta h} \delta_{0} h=0
$$

In [57] we demonstrated that for the case of a single chiral superfield, most of the structure of $\delta_{1} \Phi$ is fixed by the chiral requirement $\left(\overline{\mathrm{D}}_{\dot{\alpha}} \delta_{1} \Phi=0\right)$ and we explored the consequences of (2.4) for the choice of $S_{0}$ corresponding to the free theory of a chiral superfield. In this paper we want to explore if there are interaction terms $S_{1}$ that correspond to a different starting action $S_{0}$. 
In order to be as general as possible, we will consider as our starting point a supersymmetric nonlinear sigma model described by an arbitrary Kähler potential $\mathcal{K}(\Phi, \bar{\Phi})$ and a chiral superpotential $\mathcal{W}(\Phi)$

$$
S_{0}=\int d^{8} z \mathcal{K}(\Phi, \bar{\Phi})+\int d^{6} z \mathcal{W}(\Phi)+\int d^{6} \bar{z} \overline{\mathcal{W}}(\bar{\Phi})
$$

where the Kähler potential and the chiral superpotential are defined modulo the relations

$$
\begin{aligned}
\mathcal{K}(\Phi, \bar{\Phi}) & \sim \mathcal{K}(\Phi, \bar{\Phi})+\Lambda(\Phi)+\bar{\Lambda}(\bar{\Phi}) \\
\mathcal{W}(\Phi) & \sim \mathcal{W}(\Phi)+\text { constant }
\end{aligned}
$$

The on-shell equation of motion for this system is ${ }^{3}$

$$
\overline{\mathrm{D}}^{2} \mathcal{K}_{\Phi}=\mathcal{W}_{\Phi}
$$

and the invariance requirement (2.4) becomes:

$$
\int d^{8} z\left\{\mathcal{K}_{\Phi} \delta_{1} \Phi+\mathcal{J} \delta_{0} h\right\}+\int d^{6} z \mathcal{W}_{\Phi} \delta_{1} \Phi=0 .
$$

The above expression is symbolic in the sense that $h$ corresponds to the set of superfields that participate in the description of the $4 D, \mathcal{N}=1$ free, massless, higher spin supermultiplets and also we have assumed that the interaction terms can be written as higher spin gauge superfields times corresponding elements of the conserved supercurrent multiplet $\mathcal{J}$.

The massless, higher spin irreducible representations of the super-Poincaré group in four dimensions were first described in [65]. Later, a superfield formulation was introduced in [66-68] and further developments can be found in [69-73]. A quick synopsis of the description of higher spin supermultiplets is the following:

1. The integer superspin $Y=s(s \geq 1)$ supermultiplets $(s+1 / 2, s)^{4}$ are described by a pair of superfields $\Psi_{\alpha(s) \dot{\alpha}(s-1)}{ }^{5}$ and $V_{\alpha(s-1) \dot{\alpha}(s-1)}$ with the following zero order gauge transformations

$$
\begin{aligned}
& \delta_{0} \Psi_{\alpha(s) \dot{\alpha}(s-1)}=-\mathrm{D}^{2} L_{\alpha(s) \dot{\alpha}(s-1)}+\frac{1}{(s-1) !} \overline{\mathrm{D}}_{\left(\dot{\alpha}_{s-1}\right.} \Lambda_{\alpha(s) \dot{\alpha}(s-2))}, \\
& \delta_{0} V_{\alpha(s-1) \dot{\alpha}(s-1)}=\mathrm{D}^{\alpha_{s}} L_{\alpha(s) \dot{\alpha}(s-1)}+\overline{\mathrm{D}}^{\dot{\alpha}_{s}} \bar{L}_{\alpha(s-1) \dot{\alpha}(s)} .
\end{aligned}
$$

2. The half-integer superspin $Y=s+1 / 2$ supermultiplets $(s+1, s+1 / 2)$ have two descriptions. The first is called the transverse formulation $(s \geq 1)$ and it uses the pair of superfields $H_{\alpha(s) \dot{\alpha}(s)}, \chi_{\alpha(s) \dot{\alpha}(s-1)}$ with the following zero order gauge transformations

$$
\begin{aligned}
\delta_{0} H_{\alpha(s) \dot{\alpha}(s)} & =\frac{1}{s !} \mathrm{D}_{\left(\alpha_{s}\right.} \bar{L}_{\alpha(s-1)) \dot{\alpha}(s)}-\frac{1}{s !} \overline{\mathrm{D}}_{\left(\dot{\alpha}_{s}\right.} L_{\alpha(s) \dot{\alpha}(s-1))}, \\
\delta_{0} \chi_{\alpha(s) \dot{\alpha}(s-1)} & =\overline{\mathrm{D}}^{2} L_{\alpha(s) \dot{\alpha}(s-1)}+\mathrm{D}^{\alpha_{s+1}} \Lambda_{\alpha(s+1) \dot{\alpha}(s-1)} .
\end{aligned}
$$

\footnotetext{
${ }^{3}$ We follow the conventions of Superspace [64].

${ }^{4}$ On-shell they describe the propagation of degrees of freedom with helicity $\pm(s+1 / 2)$ and $\pm s$.

${ }^{5}$ The notation $\alpha(k)$ is a shorthand for $\mathrm{k}$ undotted symmetric indices $\alpha_{1} \alpha_{2} \ldots \alpha_{k}$. The same notation is used for the dotted indices.
} 
The second one is the longitudinal formulation $(s \geq 2)$ and it includes the superfields $H_{\alpha(s) \dot{\alpha}(s)}, \chi_{\alpha(s-1) \dot{\alpha}(s-2)}$ with

$$
\begin{aligned}
\delta_{0} H_{\alpha(s) \dot{\alpha}(s)}= & \frac{1}{s !} \mathrm{D}_{\left(\alpha_{s}\right.} \bar{L}_{\alpha(s-1)) \dot{\alpha}(s)}-\frac{1}{s !} \overline{\mathrm{D}}_{\left(\dot{\alpha}_{s}\right.} L_{\alpha(s) \dot{\alpha}(s-1))}, \\
\delta_{0} \chi_{\alpha(s-1) \dot{\alpha}(s-2)}= & \overline{\mathrm{D}}^{\dot{\alpha}_{s-1}} \mathrm{D}^{\alpha_{s}} L_{\alpha(s) \dot{\alpha}(s-1)}+\frac{s-1}{s} \mathrm{D}^{\alpha_{s}} \overline{\mathrm{D}}^{\dot{\alpha}_{s-1}} L_{\alpha(s) \dot{\alpha}(s-1)} \\
& +\frac{1}{(s-2) !} \overline{\mathrm{D}}_{\left(\dot{\alpha}_{s-2}\right.} J_{\alpha(s-1) \dot{\alpha}(s-3))} .
\end{aligned}
$$

The $s=0$ case corresponds to the well known vector supermultiplet $(1,1 / 2)$ which is being described by a real scalar superfield $V$ with the gauge transformation $\delta_{0} V=\overline{\mathrm{D}}^{2} L+\mathrm{D}^{2} \bar{L}$. The invariance of the action up to first order in $g$ as expressed in (2.4) makes obvious that if we go on-shell $\left(\frac{\delta S_{0}}{\delta \Phi}=0\right)$ we get a conservation condition on the supercurrent multiplet $\mathcal{J}$ which is controlled by the zeroth order gauge transformation of the higher spin superfields. Using the expressions above, we find the precise conservation conditions:

1. For integer superspin $Y=s \quad(s+1 / 2, s)$ we must have

$$
\mathrm{D}^{2} \overline{\mathrm{D}}^{\dot{\alpha}_{s}} \mathcal{J}_{\alpha(s) \dot{\alpha}(s)}=\frac{1}{s !} \mathrm{D}_{\left(\alpha_{s}\right.} \mathcal{T}_{\alpha(s-1)) \dot{\alpha}(s-1)}, \quad \mathcal{T}_{\alpha(s-1) \dot{\alpha}(s-1)}=\overline{\mathcal{T}}_{\alpha(s-1) \dot{\alpha}(s-1)} .
$$

2. For transverse half-integer superspin $Y=s+1 / 2 \quad(s+1, s+1 / 2)$

$$
\overline{\mathrm{D}}^{\dot{\alpha}_{s}} \mathcal{J}_{\alpha(s) \dot{\alpha}(s)}=\frac{1}{s !} \overline{\mathrm{D}}^{2} \mathrm{D}_{\left(\alpha_{s}\right.} \mathcal{T}_{\alpha(s-1)) \dot{\alpha}(s-1)}, \quad \mathcal{J}_{\alpha(s) \dot{\alpha}(s)}=\overline{\mathcal{J}}_{\alpha(s) \dot{\alpha}(s)}
$$

3. For longitudinal half-integer superspin $Y=s+1 / 2 \quad(s+1, s+1 / 2)$

$$
\begin{aligned}
\overline{\mathrm{D}}^{\dot{\alpha}_{s}} \mathcal{J}_{\alpha(s) \dot{\alpha}(s)} & =\frac{1}{s !} \mathrm{D}_{\left(\alpha_{s}\right.} \overline{\mathrm{D}}^{2} \mathcal{T}_{\alpha(s-1)) \dot{\alpha}(s-1)}-\frac{s-1}{s ! s !} \overline{\mathrm{D}}_{\left(\dot{\alpha}_{s-1}\right.} \mathrm{D}_{\left(\alpha_{s}\right.} \overline{\mathrm{D}}^{\dot{\beta}} \mathcal{T}_{\alpha(s-1)) \dot{\beta} \dot{\alpha}(s-2))}, \\
\mathcal{J}_{\alpha(s) \dot{\alpha}(s)} & =\overline{\mathcal{J}}_{\alpha(s) \dot{\alpha}(s)} .
\end{aligned}
$$

The superfields $\mathcal{J}$ and $\mathcal{T}$ (with appropriate index structures) are the higher spin supercurrent and higher spin supertrace respectively and together they define the supercurrent multiplet which generate the first order interaction terms with the higher spin gauge superfields:

1. For integer superspin $Y=s \quad(s+1 / 2, s)$

$$
\begin{aligned}
S_{1} \sim \int d^{8} z\{ & \Psi^{\alpha(s) \dot{\alpha}(s-1)} \mathcal{J}_{\alpha(s) \dot{\alpha}(s-1)}+\bar{\Psi}^{\alpha(s-1) \dot{\alpha}(s)} \overline{\mathcal{J}}_{\alpha(s-1) \dot{\alpha}(s)} \\
& \left.+V^{\alpha(s-1) \dot{\alpha}(s-1)} \mathcal{T}_{\alpha(s-1) \dot{\alpha}(s-1)}\right\}
\end{aligned}
$$

2. For transverse half-integer superspin $Y=s+1 / 2 \quad(s+1, s+1 / 2)$

$$
\begin{aligned}
S_{1} \sim \int d^{8} z & \left\{H^{\alpha(s) \dot{\alpha}(s)} \mathcal{J}_{\alpha(s) \dot{\alpha}(s)}+\chi^{\alpha(s) \dot{\alpha}(s-1)} \mathrm{D}_{\alpha_{s}} \mathcal{T}_{\alpha(s-1) \dot{\alpha}(s-1)}\right. \\
+ & \left.\bar{\chi}^{\alpha(s-1) \dot{\alpha}(s)} \overline{\mathrm{D}}_{\dot{\alpha}_{s}} \overline{\mathcal{T}}_{\alpha(s-1) \dot{\alpha}(s-1)}\right\} .
\end{aligned}
$$


3. For longitudinal half-integer superspin $Y=s+1 / 2 \quad(s+1, s+1 / 2)$

$$
\begin{aligned}
S_{1} \sim \int d^{8} z & \left\{H^{\alpha(s) \dot{\alpha}(s)} \mathcal{J}_{\alpha(s) \dot{\alpha}(s)}+\chi^{\alpha(s-1) \dot{\alpha}(s-2)} \overline{\mathrm{D}}^{\dot{\alpha}_{s-1}} \mathcal{T}_{\alpha(s-1) \dot{\alpha}(s-1)}\right. \\
+ & \left.\bar{\chi}^{\alpha(s-2) \dot{\alpha}(s-1)} \mathrm{D}^{\alpha_{s-1}} \overline{\mathcal{T}}_{\alpha(s-1) \dot{\alpha}(s-1)}\right\} .
\end{aligned}
$$

Furthermore, conservation equations (2.14) and (2.15) are not independent. They are related via an improvement term $X_{\alpha(s-1) \dot{\alpha}(s-1)}$. It is straight forward to show that if the superfields $\mathcal{J}_{\alpha(s) \dot{\alpha}(s)}, \mathcal{T}_{\alpha(s-1) \dot{\alpha}(s-1)}^{\perp}$ and $\mathcal{T}_{\alpha(s-1) \dot{\alpha}(s-1)}^{\|}$satisfy the following conservation equation

$$
\begin{aligned}
\overline{\mathrm{D}}^{\dot{\alpha}_{s}} \mathcal{J}_{\alpha(s) \dot{\alpha}(s)}= & \frac{1}{s !} \overline{\mathrm{D}}^{2} \mathrm{D}_{\left(\alpha_{s}\right.} \mathcal{T}_{\alpha(s-1)) \dot{\alpha}(s-1)}^{\perp} \\
& +\frac{1}{s !} \mathrm{D}_{\left(\alpha_{s}\right.} \overline{\mathrm{D}}^{2} \mathcal{T}_{\alpha(s-1)) \dot{\alpha}(s-1)}^{\|}-\frac{s-1}{s ! s !} \overline{\mathrm{D}}_{\left(\dot{\alpha}_{s-1}\right.} \mathrm{D}_{\left(\alpha_{s}\right.} \overline{\mathrm{D}}^{\dot{\beta}} \mathcal{T}_{\alpha(s-1)) \dot{\beta} \dot{\alpha}(s-2))}^{\|}
\end{aligned}
$$

then the hatted superfields

$$
\begin{aligned}
\hat{\mathcal{J}}_{\alpha(s) \dot{\alpha}(s)}= & \mathcal{J}_{\alpha(s) \dot{\alpha}(s)}+\frac{1}{s ! s !} \overline{\mathrm{D}}_{\left(\dot{\alpha}_{s}\right.} \mathrm{D}_{\left(\alpha_{s}\right.} X_{\alpha(s-1)) \dot{\alpha}(s-1))} \\
& -\frac{1}{s ! s !} \mathrm{D}_{\left(\alpha_{s}\right.} \overline{\mathrm{D}}_{\left(\dot{\alpha}_{s}\right.} \bar{X}_{\alpha(s-1)) \dot{\alpha}(s-1))}, \\
\hat{\mathcal{T}}_{\alpha(s-1) \dot{\alpha}(s-1)}^{\perp}= & \mathcal{T}_{\alpha(s-1) \dot{\alpha}(s-1)}^{\perp}+\frac{s+1}{s} X_{\alpha(s-1) \dot{\alpha}(s-1)}+\bar{X}_{\alpha(s-1) \dot{\alpha}(s-1)}, \\
\hat{\mathcal{T}}_{\alpha(s-1) \dot{\alpha}(s-1)}^{\|}= & \mathcal{T}_{\alpha(s-1) \dot{\alpha}(s-1)}^{\|}+\bar{X}_{\alpha(s-1) \dot{\alpha}(s-1)},
\end{aligned}
$$

satisfy exactly the same conservation equation. So, there is a choice of $X_{\alpha(s-1) \dot{\alpha}(s-1)}$ that will convert (2.14) $\left[\mathcal{T}_{\alpha(s-1) \dot{\alpha}(s-1)}^{\|}=0\right]$ to $(2.15)\left[\hat{\mathcal{T}}_{\alpha(s-1) \dot{\alpha}(s-1)}^{\perp}=0\right]$ and another one to go from $(2.15)\left[\mathcal{T}_{\alpha(s-1) \dot{\alpha}(s-1)}^{\perp}=0\right]$ to $(2.14)\left[\hat{\mathcal{T}}_{\alpha(s-1) \dot{\alpha}(s-1)}^{\|}=0\right]$. This is a manifestation of the fact that the two formulations of half-integer superspin supermultiplets are dual to each other.

Based on the results of [57] we know that if $\delta_{1} \Phi$ is linear in derivatives of $\Phi^{1}$ then interactions with integer superspin supermultiplets require more than one chiral supefields. Therefore, in this paper we will focus our efforts in constructing interactions with half integer superspin supermultiplets $(s+1, s+1 / 2)$ of the (2.17) kind via higher spin supercurrent multiplets that satisfy conservation equation (2.14). This will be done in the following sections. However, in order to get some intuition and understand all the contributing factors we will not start with the arbitrary spin case but from $s=0$ (vector supermultiplet) to $s=1$ (supergravity) and then to higher spin supermultiplets $(s \geq 2)$. Furthermore, in order to avoid unnecessary complexity we will turn off $\mathcal{W}$ for the next three sections and consider only the effects of $\mathcal{K}$. The contributions of $\mathcal{W}$ will be examined in section six.

\section{Coupling to vector supermultiplet}

In this case, the conservation equation (2.14) gets simplified to

$$
\overline{\mathrm{D}}^{2} \mathcal{J}=0
$$


and the supercurrent multiplet has only one element, the real, scalar supercurrent $\mathcal{J}$. Due to (2.9) and the structure of $\delta_{1} \Phi$ as found in [57], the supercurrent $\mathcal{J}$ must depend on $\Phi, \bar{\Phi}$ but crucially not in their derivatives. Hence we should be able to express $\mathcal{J}$ as a power series

$$
\mathcal{J}=\sum_{p} \sum_{q} \Phi^{p} \bar{\Phi}^{q} A_{p, q}
$$

where $A_{p, q}$ are a set of constants. The conservation equation (3.1) gives:

$$
\overline{\mathrm{D}}^{2} \mathcal{J}=\sum_{p} \Phi^{p} \overline{\mathrm{D}}^{2}\left[\sum_{q} \bar{\Phi}^{q} A_{p, q}\right]=0 .
$$

Furthermore, because it must hold on-shell $\left(\overline{\mathrm{D}}^{2} \mathcal{K}_{\Phi}=0\right)$, we must have

$$
\left.\overline{\mathrm{D}}^{2}\left[\sum_{q} \bar{\Phi}^{q} A_{p, q}\right]\right|_{\overline{\mathrm{D}}^{2} \mathcal{K}_{\Phi}=0}=0 \Rightarrow \sum_{q} \bar{\Phi}^{q} A_{p, q}=f_{p}(\Phi) \mathcal{K}_{\Phi}
$$

where $f_{p}(\Phi)$ is a function of $\Phi$. Hence, we conclude that $\mathcal{J}$ must be of the form

$$
\mathcal{J}=\sum_{p} \Phi^{p} f_{p}(\Phi) \mathcal{K}_{\Phi}=F(\Phi) \mathcal{K}_{\Phi}
$$

where $F(\Phi)=\sum_{p} \Phi^{p} f_{p}(\Phi)$. However, $\mathcal{J}$ by definition has to be real therefore we must have

$$
F(\Phi) \mathcal{K}_{\Phi}=\bar{F}(\bar{\Phi}) \overline{\mathcal{K}}_{\bar{\Phi}}
$$

For $F(\Phi) \neq 0$ which is the non-trivial case we are being interested, we can define a new chiral superfield $\varphi$ as follows:

$$
\varphi=\exp \left[\int d \Phi F^{-1}(\Phi)\right] .
$$

For this new variable the on-shell equation of motion has the same form as before $\left(\overline{\mathrm{D}}^{2} \mathcal{K}_{\varphi}=\right.$ 0 ), the supercurrent $\mathcal{J}$ takes the form

$$
\mathcal{J}=\varphi \mathcal{K}_{\varphi}
$$

and the reality condition takes the simpler expression

$$
\varphi \mathcal{K}_{\varphi}=\bar{\varphi} \mathcal{K}_{\bar{\varphi}} .
$$

This can be satisfied only if $\mathcal{K}$ is a function of the product $\bar{\varphi} \varphi,{ }^{6} \mathcal{K}=\mathcal{K}(\bar{\varphi} \varphi)$. This constraint in $\mathcal{K}$ can be understood as the demand for the Kähler potential to have a global $\mathrm{U}(1)$ symmetry expressed by the phase shift of $\varphi^{7}$

$$
\varphi \rightarrow e^{i \lambda} \varphi
$$

which can be gauged in order to generate interactions with the vector supermultiplet.

\footnotetext{
${ }^{6}$ Equivalently, one can define another chiral superfield $\phi=\ln (\varphi)=\int d \Phi F^{-1}(\Phi)$ under which the Kähler potential is a function of the sum $\phi+\bar{\phi}, \mathcal{K}=\mathcal{K}(\phi+\bar{\phi})$ and the supercurrent is $\mathcal{J}=\mathcal{K}_{\phi}$. A detailed discussion of this can be found in [64] where the connection between the action of a real linear $G=\phi+\bar{\phi}$ and the action of a chiral $\varphi$ in presented.

${ }^{7}$ For the variable $\phi$ the global $\mathrm{U}(1)$ is realized as a shift symmetry $\phi \rightarrow \phi+i \lambda$.
} 


\section{Coupling to (non-minimal) supergravity}

Next, we attempt the construction of the supercurrent multiplet that generates interactions with non-minimal supergravity [transverse formulation of supermultiplet $(2,3 / 2)$ ] which satisfies the conservation equation

$$
\overline{\mathrm{D}}^{\dot{\alpha}} \mathcal{J}_{\alpha \dot{\alpha}}=\overline{\mathrm{D}}^{2} \mathrm{D}_{\alpha} \mathcal{T}
$$

For the supercurrent and supertrace we consider the following ansatz:

$$
\begin{aligned}
\mathcal{J}_{\alpha \dot{\alpha}}= & \delta \partial_{\alpha \dot{\alpha}} \Phi \mathcal{K}_{\Phi}+d \partial_{\alpha \dot{\alpha}} \bar{\Phi} \mathcal{K}_{\bar{\Phi}} \\
& +\alpha \mathrm{D}_{\alpha} \Phi \overline{\mathrm{D}}_{\dot{\alpha}} \mathcal{K}_{\Phi}-a \overline{\mathrm{D}}_{\dot{\alpha}} \bar{\Phi} \mathrm{D}_{\alpha} \mathcal{K}_{\bar{\Phi}} \\
& +\beta \Phi \mathrm{D}_{\alpha} \overline{\mathrm{D}}_{\dot{\alpha}} \mathcal{K}_{\Phi}-b \bar{\Phi} \overline{\mathrm{D}}_{\dot{\alpha}} \mathrm{D}_{\alpha} \mathcal{K}_{\bar{\Phi}} \\
& +\gamma \Phi \overline{\mathrm{D}}_{\dot{\alpha}} \mathrm{D}_{\alpha} \mathcal{K}_{\Phi}-c \bar{\Phi} \mathrm{D}_{\alpha} \overline{\mathrm{D}}_{\dot{\alpha}} \mathcal{K}_{\bar{\Phi}} \\
\mathcal{T}= & e \mathcal{K}+\kappa \Phi \mathcal{K}_{\Phi}+h \bar{\Phi} \mathcal{K}_{\bar{\Phi}} .
\end{aligned}
$$

\subsection{Improvement terms}

The definition of the supercurrent multiplet $\left(\mathcal{J}_{\alpha \dot{\alpha}}, \mathcal{T}\right)$ via conservation equation (4.1) is not unique. There are improvement terms that one should consider. In this case, there is an arbitrary superfield $U_{\alpha}$ such that the supercurrent multiplet $\left(\hat{\mathcal{J}}_{\alpha \dot{\alpha}}, \hat{\mathcal{T}}\right)$ defined by:

$$
\begin{aligned}
\hat{\mathcal{J}}_{\alpha \dot{\alpha}} & =\mathcal{J}_{\alpha \dot{\alpha}}+\mathrm{D}_{\alpha} \overline{\mathrm{D}}^{2} \bar{U}_{\dot{\alpha}}-\overline{\mathrm{D}}_{\dot{\alpha}} \mathrm{D}^{2} U_{\alpha}, \\
\hat{\mathcal{T}} & =\mathcal{T}+2 \mathrm{D}^{\alpha} U_{\alpha}+\overline{\mathrm{D}}^{\dot{\alpha}} \bar{U}_{\dot{\alpha}}
\end{aligned}
$$

satisfies the same conservation equation (4.1). This can also be extracted from (2.19), (2.20a) and the demand that the hat supercurrent and supertrace stay in the transverse formulation. If we select

$$
U_{\alpha}=r \mathrm{D}_{\alpha} \bar{\Lambda} \mathcal{K}_{\bar{\Phi}}
$$

where $\Lambda$ is the prepotential of chiral superfield $\Phi=\overline{\mathrm{D}}^{2} \Lambda$, then the $r$ parameter can be used to eliminate $b$ in (4.2). Hence the ansatz for $\mathcal{J}_{\alpha \dot{\alpha}}$ becomes

$$
\begin{aligned}
\mathcal{J}_{\alpha \dot{\alpha}}= & \delta \partial_{\alpha \dot{\alpha}} \Phi \mathcal{K}_{\Phi}+d \partial_{\alpha \dot{\alpha}} \bar{\Phi} \mathcal{K}_{\bar{\Phi}} \\
& +\alpha \mathrm{D}_{\alpha} \Phi \overline{\mathrm{D}}_{\dot{\alpha}} \mathcal{K}_{\Phi}-a \overline{\mathrm{D}}_{\dot{\alpha}} \bar{\Phi} \mathrm{D}_{\alpha} \mathcal{K}_{\bar{\Phi}} \\
& +\beta \Phi \mathrm{D}_{\alpha} \overline{\mathrm{D}}_{\dot{\alpha}} \mathcal{K}_{\Phi} \\
& +\gamma \Phi \overline{\mathrm{D}}_{\dot{\alpha}} \mathrm{D}_{\alpha} \mathcal{K}_{\Phi}-c \bar{\Phi} \mathrm{D}_{\alpha} \overline{\mathrm{D}}_{\dot{\alpha}} \mathcal{K}_{\bar{\Phi}}
\end{aligned}
$$

\subsection{Conservation equation}

Now we use (4.6) and (4.3) to determine the consequences of conservation equation (4.1). The result is:

$$
\begin{aligned}
0=\overline{\mathrm{D}}^{\dot{\alpha}} \mathcal{J}_{\alpha \dot{\alpha}}-\overline{\mathrm{D}}^{2} \mathrm{D}_{\alpha} \mathcal{T}= & (i \delta+\alpha+\kappa+e) \overline{\mathrm{D}}^{\dot{\alpha}} \mathrm{D}_{\alpha} \Phi \overline{\mathrm{D}}_{\dot{\alpha}} \mathcal{K}_{\Phi}+(2 \gamma-\beta-\kappa) \Phi \overline{\mathrm{D}}^{2} \mathrm{D}_{\alpha} \mathcal{K}_{\Phi} \\
& +i d \mathrm{D}_{\alpha} \overline{\mathrm{D}}^{2} \bar{\Phi} \mathcal{K}_{\bar{\Phi}}+i d \mathrm{D}_{\alpha} \overline{\mathrm{D}}^{\dot{\alpha}} \bar{\Phi} \overline{\mathrm{D}}_{\dot{\alpha}} \mathcal{K}_{\bar{\Phi}} \\
& -(2 a+h) \overline{\mathrm{D}}^{2} \bar{\Phi} \mathrm{D}_{\alpha} \mathcal{K}_{\bar{\Phi}}-(a+h) \overline{\mathrm{D}}^{\dot{\alpha}} \bar{\Phi} \overline{\mathrm{D}}_{\dot{\alpha}} \mathrm{D}_{\alpha} \mathcal{K}_{\bar{\Phi}} \\
& -c \overline{\mathrm{D}}^{\dot{\alpha}} \bar{\Phi} \mathrm{D}_{\alpha} \overline{\mathrm{D}}_{\dot{\alpha}} \mathcal{K}_{\bar{\Phi}}-(h-c) \bar{\Phi} \overline{\mathrm{D}}^{2} \mathrm{D}_{\alpha} \mathcal{K}_{\bar{\Phi}} \\
& +c \bar{\Phi} \mathrm{D}_{\alpha} \overline{\mathrm{D}}^{2} \mathcal{K}_{\bar{\Phi}}
\end{aligned}
$$


Assuming that the Kähler potential $\mathcal{K}$ is arbitrary, meaning it does not have special properties that relate some of the terms above with each other and they are independent, we conclude that the coefficient of each term must vanish:

$$
\begin{aligned}
e & =-i \delta-\alpha-2 \gamma+\beta, \\
\kappa & =2 \gamma-\beta, \\
a & =0 \\
c & =0 \\
d & =0 \\
h & =0
\end{aligned}
$$

and the expressions for the conserved $\mathcal{J}_{\alpha \dot{\alpha}}$ and $\mathcal{T}$ are:

$$
\begin{aligned}
\mathcal{J}_{\alpha \dot{\alpha}} & =\delta \partial_{\alpha \dot{\alpha}} \Phi \mathcal{K}_{\Phi}+\alpha \mathrm{D}_{\alpha} \Phi \overline{\mathrm{D}}_{\dot{\alpha}} \mathcal{K}_{\Phi}+\beta \Phi \mathrm{D}_{\alpha} \overline{\mathrm{D}}_{\dot{\alpha}} \mathcal{K}_{\Phi}+\gamma \Phi \overline{\mathrm{D}}_{\dot{\alpha}} \mathrm{D}_{\alpha} \mathcal{K}_{\Phi} \\
\mathcal{T} & =(-i \delta-\alpha-2 \gamma+\beta) \mathcal{K}+(2 \gamma-\beta) \Phi \mathcal{K}_{\Phi} .
\end{aligned}
$$

\subsection{Reality condition}

The last condition we must impose is the reality of $\mathcal{J}_{\alpha \dot{\alpha}}$. For this it will be useful to take into account the following expressions

$$
\begin{aligned}
\overline{\mathrm{D}}_{\dot{\alpha}} \mathcal{K}_{\Phi} & =\overline{\mathrm{D}}_{\dot{\alpha}} \bar{\Phi} \mathcal{K}_{\Phi \bar{\Phi}} \\
\mathrm{D}_{\alpha} \overline{\mathrm{D}}_{\dot{\alpha}} \mathcal{K}_{\Phi} & =i \partial_{\alpha \dot{\alpha}} \bar{\Phi} \mathcal{K}_{\Phi \bar{\Phi}}+\mathrm{D}_{\alpha} \Phi \overline{\mathrm{D}}_{\dot{\alpha}} \bar{\Phi} \mathcal{K}_{\Phi \Phi \bar{\Phi}} \\
\overline{\mathrm{D}}_{\dot{\alpha}} \mathrm{D}_{\alpha} \mathcal{K}_{\Phi} & =i \partial_{\alpha \dot{\alpha}} \Phi \mathcal{K}_{\Phi \Phi}-\mathrm{D}_{\alpha} \Phi \overline{\mathrm{D}}_{\dot{\alpha}} \bar{\Phi} \mathcal{K}_{\Phi \Phi \bar{\Phi}}
\end{aligned}
$$

which can be used to re-write (4.9) in the following manner

$$
\begin{aligned}
\mathcal{J}_{\alpha \dot{\alpha}}= & \partial_{\alpha \dot{\alpha}} \Phi\left[\delta \mathcal{K}_{\Phi}+i \gamma \Phi \mathcal{K}_{\Phi \Phi}\right] \\
& +\partial_{\alpha \dot{\alpha}} \bar{\Phi}\left[i \beta \Phi \mathcal{K}_{\Phi \bar{\Phi}}\right] \\
& +\mathrm{D}_{\alpha} \Phi \overline{\mathrm{D}}_{\dot{\alpha}} \bar{\Phi}\left[\alpha \mathcal{K}_{\Phi \bar{\Phi}}+(\beta-\gamma) \Phi \mathcal{K}_{\Phi \Phi \bar{\Phi}}\right]
\end{aligned}
$$

The reality of $\mathcal{J}_{\alpha \dot{\alpha}}$ as expressed above demands:

$$
\begin{array}{r}
\delta \mathcal{K}_{\Phi}+i \gamma \Phi \mathcal{K}_{\Phi \Phi}+i \beta^{*} \bar{\Phi} \mathcal{K}_{\Phi \bar{\Phi}}=0, \\
\text { 2.) } \quad\left(\alpha^{*}-\alpha\right) \mathcal{K}_{\Phi \bar{\Phi}}-(\beta-\gamma) \Phi \mathcal{K}_{\Phi \Phi \bar{\Phi}}+(\beta-\gamma)^{*} \bar{\Phi} \mathcal{K}_{\Phi \bar{\Phi} \bar{\Phi}}=0 .
\end{array}
$$

For an arbitrary Kähler potential, the above constraints can be satisfied only if

$$
\alpha=\alpha^{*}, \beta=\gamma=\delta=0 .
$$

Therefore, we conclude that for an arbitrary Kähler potential $\mathcal{K}$ there exist a supercurrent multiplet

$$
\begin{aligned}
\mathcal{J}_{\alpha \dot{\alpha}} & =\mathrm{D}_{\alpha} \Phi \overline{\mathrm{D}}_{\dot{\alpha}} \mathcal{K}_{\Phi} \\
\mathcal{T} & =-\mathcal{K}
\end{aligned}
$$


Conceptually, this result was to be expected because we know that any theory can be coupled to supergravity. Expressions, (4.16) and (4.17) give the supercurrent multiplet that generates the linearized interaction (first order in $g$ ) between a supersymmetric nonlinear model of a single chiral superfield described by Kähler potential $\mathcal{K}$ and non-minimal supergravity. Additionally, we observe that for arbitrary $\mathcal{K}$ the supertrace $\mathcal{T}$ is not zero and for that case the supercurrent multiplet defined by $\left\{\mathcal{J}_{\alpha \dot{\alpha}}, \mathcal{T}\right\}$ is canonical $[57,63]$. However, it is straight forward to see that if the Kähler potential has the property

$$
\mathcal{K} \sim \Phi \mathcal{K}_{\Phi}
$$

then there is an improvement term of type (4.5) such that the improved supertrace $\hat{\mathcal{T}}$ (4.4a) will be zero

$$
\hat{\mathcal{T}}=0
$$

and the new supercurrent multiplet $\left\{\hat{\mathcal{J}}_{\alpha \dot{\alpha}}, 0\right\}$ is a minimal one. This is possible only if the Kähler potential is a function of the product $\bar{\Phi} \Phi, \quad \mathcal{K}=\mathcal{K}(\bar{\Phi} \Phi)$, which includes the free theory. Furthermore, by converting (4.16) and (4.17) via (2.20a) to the longitudinal formulation of supergravity (minimal supergravity) we recover the results of [63].

\section{Coupling to higher spin supermultiplets}

Based on the two previous lower spin examples we build confidence on the workings of our method and it is time to generalize it to higher spin supermultiplet $(s+1, s+1 / 2)$ with $s \geq 2$. For this case the conservation equation we must satisfy is (2.14) and we write the following ansatz for the supercurrent and the supertrace: ${ }^{8}$

$$
\begin{aligned}
\mathcal{J}_{\alpha(s) \dot{\alpha}(s)}= & \delta \partial^{(s)} \Phi \mathcal{K}_{\Phi}+d \partial^{(s)} \bar{\Phi} \mathcal{K}_{\bar{\Phi}} \\
& +\sum_{p=0}^{s-1} \alpha_{p} \partial^{(p)} \mathrm{D} \Phi \partial^{(s-p-1)} \overline{\mathrm{D}} \mathcal{K}_{\Phi}-\sum_{p=0}^{s-1} a_{p} \partial^{(p)} \overline{\mathrm{D}} \bar{\Phi} \partial^{(s-p-1)} \mathrm{D} \mathcal{K}_{\bar{\Phi}} \\
& +\sum_{p=0}^{s-1} \beta_{p} \partial^{(p)} \Phi \partial^{(s-p-1)} \mathrm{D} \overline{\mathrm{D}} \mathcal{K}_{\Phi}-\sum_{p=0}^{s-1} b_{p} \partial^{(p)} \bar{\Phi} \partial^{(s-p-1)} \overline{\mathrm{D}} \mathrm{D} \mathcal{K}_{\bar{\Phi}} \\
& +\sum_{p=0}^{s-1} \gamma_{p} \partial^{(p)} \Phi \partial^{(s-p-1)} \overline{\mathrm{D}} \mathrm{D} \mathcal{K}_{\Phi}-\sum_{p=0}^{s-1} c_{p} \partial^{(p)} \bar{\Phi} \partial^{(s-p-1)} \mathrm{D} \overline{\mathrm{D}} \mathcal{K}_{\bar{\Phi}}, \\
\mathcal{T}_{\alpha(s-1) \dot{\alpha}(s-1)} & e \partial^{(s-1)} \mathcal{K}+\kappa \partial^{(s-1)} \Phi \mathcal{K}_{\Phi}+h \partial^{(s-1)} \bar{\Phi} \mathcal{K}_{\bar{\Phi}} \\
& +\sum_{p=0}^{s-2} \ell_{p} \partial^{(p)} \mathrm{D} \Phi \partial^{(s-p-2)} \overline{\mathrm{D}} \mathcal{K}_{\Phi}+\sum_{p=0}^{s-2} f_{p} \partial^{(p)} \overline{\mathrm{D}} \bar{\Phi} \partial^{(s-p-2)} \mathrm{D} \mathcal{K}_{\bar{\Phi}} \\
& +\sum_{p=0}^{s-2} \zeta_{p} \partial^{(p)} \Phi \partial^{(s-p-2)} \overline{\mathrm{D}} \mathrm{D} \mathcal{K}_{\Phi}+\sum_{p=0}^{s-2} g_{p} \partial^{(p)} \bar{\Phi} \partial^{(s-p-2)} \overline{\mathrm{D}} \mathrm{D} \mathcal{K}_{\bar{\Phi}} \\
& +\sum_{p=0}^{s-2} \xi_{p} \partial^{(p)} \Phi \partial^{(s-p-2)} \mathrm{D} \overline{\mathrm{D}} \mathcal{K}_{\Phi}+\sum_{p=0}^{s-2} s_{p} \partial^{(p)} \bar{\Phi} \partial^{(s-p-2)} \mathrm{D} \overline{\mathrm{D}} \mathcal{K}_{\bar{\Phi}} .
\end{aligned}
$$

\footnotetext{
${ }^{8}$ For simplicity, we omit to write explicitly the free indices and their symmetrization when necessary. Also the symbol $\partial^{(p)}$ is an abbreviation for a string of $p$ spacetime derivatives.
} 
However, because $s \geq 2$, the first term of $\mathcal{T}_{\alpha(s-1) \dot{\alpha}(s-1)}$ can be expanded in the following manner:

$$
\begin{aligned}
\partial^{(s-1)} \mathcal{K}= & \partial^{(s-2)}\left(\partial \Phi \mathcal{K}_{\Phi}+\partial \bar{\Phi} \mathcal{K}_{\bar{\Phi}}\right) \\
= & \partial^{(s-1)} \Phi \mathcal{K}_{\Phi}+\partial^{(s-1)} \bar{\Phi} \mathcal{K}_{\bar{\Phi}} \\
& -i \sum_{p=1}^{s-2}\left(\begin{array}{c}
s-2 \\
p-1
\end{array}\right) \partial^{(p)} \Phi \partial^{(s-p-2)}(\mathrm{D} \overline{\mathrm{D}}+\overline{\mathrm{D}} \mathrm{D}) \mathcal{K}_{\Phi} \\
& -i \sum_{p=1}^{s-2}\left(\begin{array}{c}
s-2 \\
p-1
\end{array}\right) \partial^{(p)} \bar{\Phi} \partial^{(s-p-2)}(\mathrm{D} \overline{\mathrm{D}}+\overline{\mathrm{D}} \mathrm{D}) \mathcal{K}_{\bar{\Phi}}
\end{aligned}
$$

Hence, this term is not independent any more, as in the supergravity case, and all it does is to redefine the $\kappa, h, \zeta_{p}, g_{p}, \xi_{p}$ and $s_{p}$ coefficients. Therefore, we can ignore it $(e=0)$. Moreover, both $\mathcal{J}_{\alpha(s) \dot{\alpha}(s)}$ and $\mathcal{T}_{\alpha(s-1) \dot{\alpha}(s-1)}$ are not uniquely defined but up to an equivalence class. This is due to the presence of terms that identically vanish in both left and right hand sides of (2.14) due to the D algebra. For $\mathcal{T}_{\alpha(s-1) \dot{\alpha}(s-1)}$ this equivalence relation is the following:

$$
\begin{aligned}
\mathcal{T}_{\alpha(s-1) \dot{\alpha}(s-1)} \sim & \mathcal{T}_{\alpha(s-1) \dot{\alpha}(s-1)}+\frac{1}{(s-1) !} \mathrm{D}_{\left(\alpha_{s-1}\right.} P_{\alpha(s-2)) \dot{\alpha}(s-1)}^{(1)} \\
& +\mathrm{D}^{2} P_{\alpha(s-1) \dot{\alpha}(s-1)}^{(2)}+\overline{\mathrm{D}}^{2} P_{\alpha(s-1) \dot{\alpha}(s-1)}^{(3)}
\end{aligned}
$$

for arbitrary superfields $P_{\alpha(s-2) \dot{\alpha}(s-1)}^{(1)}, P_{\alpha(s-1) \dot{\alpha}(s-1)}^{(2)}, P_{\alpha(s-1) \dot{\alpha}(s-1)}^{(3)}$. This means that we can immediately ignore the $\ell_{p}$ terms because they can be converted to $\xi_{p}$ terms. Also, the $f_{p}$ terms can be ignored because they can be converted to $g_{p}$ and $s_{p}$ terms and additionally all the $s_{p}$ terms can be disregarded. Therefore, the ansatz for the higher spin supertrace takes the form:

$$
\begin{aligned}
\mathcal{T}_{\alpha(s-1) \dot{\alpha}(s-1)}= & \kappa \partial^{(s-1)} \Phi \mathcal{K}_{\Phi}+h \partial^{(s-1)} \bar{\Phi} \mathcal{K}_{\bar{\Phi}} \\
& +\sum_{p=0}^{s-2} \zeta_{p} \partial^{(p)} \Phi \partial^{(s-p-2)} \overline{\mathrm{D}} \mathrm{D} \mathcal{K}_{\Phi}+\sum_{p=0}^{s-2} g_{p} \partial^{(p)} \bar{\Phi} \partial^{(s-p-2)} \overline{\mathrm{D}} \mathrm{D} \mathcal{K}_{\bar{\Phi}} \\
& +\sum_{p=0}^{s-2} \xi_{p} \partial^{(p)} \Phi \partial^{(s-p-2)} \mathrm{D} \overline{\mathrm{D}} \mathcal{K}_{\Phi}
\end{aligned}
$$

\subsection{Improvement terms}

Now we consider various improvement terms that will further reduce the unknown parameters in the above expressions. The arguments that led to (4.4a) also hold for $s \geq 2$ as well. Thus, the improvement terms we have are parametrized by an unconstrained superfield $U_{\alpha(s) \dot{\alpha}(s-1)}$ and define the improved supercurrent and supertrace as follows:

$$
\begin{aligned}
\hat{\mathcal{J}}_{\alpha(s) \dot{\alpha}(s)} & =\mathcal{J}_{\alpha(s) \dot{\alpha}(s)}+\frac{1}{s !} \mathrm{D}_{\left(\alpha_{s}\right.} \overline{\mathrm{D}}^{2} \bar{U}_{\alpha(s-1)) \dot{\alpha}(s)}-\frac{1}{s !} \overline{\mathrm{D}}_{\left(\dot{\alpha}_{s}\right.} \mathrm{D}^{2} U_{\alpha(s) \dot{\alpha}(s-1))}, \\
\hat{\mathcal{T}}_{\alpha(s-1) \dot{\alpha}(s-1)} & =\mathcal{T}_{\alpha(s-1) \dot{\alpha}(s-1)}+\frac{s+1}{s} \mathrm{D}^{\alpha_{s}} U_{\alpha(s) \dot{\alpha}(s-1)}+\overline{\mathrm{D}}^{\dot{\alpha}_{s}} \bar{U}_{\alpha(s-1) \dot{\alpha}(s)} .
\end{aligned}
$$


One can show that if we select $U_{\alpha(s) \dot{\alpha}(s-1)}$ in the following way

$$
\begin{aligned}
U_{\alpha(s) \dot{\alpha}(s-1)}= & r \partial^{(s-1)} \mathrm{D} \bar{\Lambda} \mathcal{K}_{\bar{\Phi}} \\
& +\sum_{p=0}^{s-2} \rho_{p} \partial^{(p)} \mathrm{D} \bar{\Lambda} \partial^{(s-p-2)} \overline{\mathrm{D}} \mathrm{D} \mathcal{K}_{\bar{\Phi}} \\
& +\sum_{p=0}^{s-2} \sigma_{p} \partial^{(p)} \mathrm{D} \bar{\Lambda} \partial^{(s-p-2)} \mathrm{D} \overline{\mathrm{D}} \mathcal{K}_{\bar{\Phi}}
\end{aligned}
$$

for some parameters $r, \rho_{p}, \sigma_{p}$, then ${ }^{9}$

$$
\begin{aligned}
\hat{\mathcal{J}}_{\alpha(s) \dot{\alpha}(s)}-\mathcal{J}_{\alpha(s) \dot{\alpha}(s)}= & r \partial^{(s-1)} \bar{\Phi} \overline{\mathrm{D}} \mathrm{D} \mathcal{K}_{\bar{\Phi}}-r^{*} \partial^{(s-1)} \Phi \mathrm{D} \overline{\mathrm{D}} \mathcal{K}_{\Phi} \\
& +\sum_{p=0}^{s-2} i \rho_{p} \partial^{(p)} \bar{\Phi} \partial^{(s-p-1)} \overline{\mathrm{D}} \mathrm{D} \mathcal{K}_{\bar{\Phi}}+\sum_{p=0}^{s-2} i \rho_{p}^{*} \partial^{(p)} \Phi \partial^{(s-p-1)} \mathrm{D} \overline{\mathrm{D}} \mathcal{K}_{\Phi} \\
& +r \partial^{(s-1)} \overline{\mathrm{D}} \bar{\Phi} \mathrm{D} \mathcal{K}_{\bar{\Phi}}-r^{*} \partial^{(s-1)} \mathrm{D} \Phi \overline{\mathrm{D}} \mathcal{K}_{\Phi} \\
& +\sum_{p=0}^{s-2} i \rho_{p} \partial^{(p)} \overline{\mathrm{D}} \bar{\Phi} \partial^{(s-p-1)} \mathrm{D} \mathcal{K}_{\bar{\Phi}}+\sum_{p=0}^{s-2} i \rho_{p}^{*} \partial^{(p)} \mathrm{D} \Phi \partial^{(s-p-1)} \overline{\mathrm{D}} \mathcal{K}_{\Phi}
\end{aligned}
$$

As a result, we can select parameters $r$ and $\rho_{p}$ in order to eliminate the $b_{p}$ terms in (5.1). Thus we get

$$
\begin{aligned}
\mathcal{J}_{\alpha(s) \dot{\alpha}(s)}= & \delta \partial^{(s)} \Phi \mathcal{K}_{\Phi}+d \partial^{(s)} \bar{\Phi} \mathcal{K}_{\bar{\Phi}} \\
& +\sum_{p=0}^{s-1} \alpha_{p} \partial^{(p)} \mathrm{D} \Phi \partial^{(s-p-1)} \overline{\mathrm{D}} \mathcal{K}_{\Phi}-\sum_{p=0}^{s-1} a_{p} \partial^{(p)} \overline{\mathrm{D}} \bar{\Phi} \partial^{(s-p-1)} \mathrm{D} \mathcal{K}_{\bar{\Phi}} \\
& +\sum_{p=0}^{s-1} \beta_{p} \partial^{(p)} \Phi \partial^{(s-p-1)} \mathrm{D} \overline{\mathrm{D}} \mathcal{K}_{\Phi} \\
& +\sum_{p=0}^{s-1} \gamma_{p} \partial^{(p)} \Phi \partial^{(s-p-1)} \overline{\mathrm{D}} \mathrm{D} \mathcal{K}_{\Phi}-\sum_{p=0}^{s-1} c_{p} \partial^{(p)} \bar{\Phi} \partial^{(s-p-1)} \mathrm{D} \overline{\mathrm{D}} \mathcal{K}_{\bar{\Phi}}
\end{aligned}
$$

\subsection{Additional freedom}

For $s \geq 2$ there is some additional freedom in defining the higher spin supercurrent and supertrace. Let us consider the following quantity:

$$
Z_{\alpha(s-1) \dot{\alpha}(s-1)}=\partial^{(s-2)}\left(\partial \bar{\Phi} \mathcal{K}_{\bar{\Phi}}+i \Phi \mathrm{D} \overline{\mathrm{D}} \mathcal{K}_{\Phi}\right)
$$

It is straight forward to prove that

$$
\mathrm{D}_{\left(\alpha_{s}\right.} Z_{\alpha(s-1)) \dot{\alpha}(s-1)}=i \partial^{(s-2)}\left(\mathrm{D} \Phi \mathrm{D} \Phi \overline{\mathrm{D}} \bar{\Phi} \mathcal{K}_{\Phi \Phi \bar{\Phi}}\right)=0 .
$$

\footnotetext{
${ }^{9}$ Notice that the $\sigma_{p}$ terms do not participate in the result. That is because they describe the freedom in the definition of $U_{\alpha(s) \dot{\alpha}(s-1)}$.
} 
It vanishes due to the symmetrization of the two $\mathrm{D} \Phi$ terms. Hence, we can enhance the equivalence class in the definition of $\mathcal{J}_{\alpha(s) \dot{\alpha}(s)}$ and $\mathcal{T}_{\alpha(s-1) \dot{\alpha}(s-1)}$ by adding the following terms

$$
\begin{aligned}
& \mathcal{T}_{\alpha(s-1) \dot{\alpha}(s-1)} \sim \mathcal{T}_{\alpha(s-1) \dot{\alpha}(s-1)}+c_{1} Z_{\alpha(s-1) \dot{\alpha}(s-1)}, \\
& \mathcal{J}_{\alpha(s) \dot{\alpha}(s)} \sim \mathcal{J}_{\alpha(s) \dot{\alpha}(s)}+c_{2} \overline{\mathrm{D}}_{\left(\dot{\alpha}_{s}\right.} \mathrm{D}_{\left(\alpha_{s}\right.} Z_{\alpha(s-1)) \dot{\alpha}(s-1)}+c_{3} \mathrm{D}_{\left(\alpha_{s}\right.} \overline{\mathrm{D}}_{\left(\dot{\alpha}_{s}\right.} \bar{Z}_{\alpha(s-1)) \dot{\alpha}(s-1)} \cdot
\end{aligned}
$$

Because the $c_{2}$ and $c_{3}$ terms are identically zero they do not change $\mathcal{J}_{\alpha(s) \dot{\alpha}(s)}$, whereas the $c_{1}$ term is not zero but does not contribute in the conservation equation. If we expand these terms in a manner similar to (5.3) we get that the $h$ term in (5.5) can be set to zero by $c_{1}$, the $\alpha_{s-1}$ term in (5.9) can be set to zero by $c_{3}$ and similarly the $a_{s-1}$ term by $c_{2}$. Hence, we should consider the following expressions for the higher spin supercurrent multiplet:

$$
\begin{aligned}
\mathcal{J}_{\alpha(s) \dot{\alpha}(s)}= & \delta \partial^{(s)} \Phi \mathcal{K}_{\Phi}+d \partial^{(s)} \bar{\Phi} \mathcal{K}_{\bar{\Phi}} \\
& +\sum_{p=0}^{s-2} \alpha_{p} \partial^{(p)} \mathrm{D} \Phi \partial^{(s-p-1)} \overline{\mathrm{D}} \mathcal{K}_{\Phi}-\sum_{p=0}^{s-2} a_{p} \partial^{(p)} \overline{\mathrm{D}} \bar{\Phi} \partial^{(s-p-1)} \mathrm{D} \mathcal{K}_{\bar{\Phi}} \\
& +\sum_{p=0}^{s-1} \beta_{p} \partial^{(p)} \Phi \partial^{(s-p-1)} \mathrm{D} \overline{\mathrm{D}} \mathcal{K}_{\Phi} \\
& +\sum_{p=0}^{s-1} \gamma_{p} \partial^{(p)} \Phi \partial^{(s-p-1)} \overline{\mathrm{D}} \mathrm{D} \mathcal{K}_{\Phi}-\sum_{p=0}^{s-1} c_{p} \partial^{(p)} \bar{\Phi} \partial^{(s-p-1)} \mathrm{D} \overline{\mathrm{D}} \mathcal{K}_{\bar{\Phi}} \\
\mathcal{T}_{\alpha(s-1) \dot{\alpha}(s-1)} & \kappa \partial^{(s-1)} \Phi \mathcal{K}_{\Phi} \\
& +\sum_{p=0}^{s-2} \zeta_{p} \partial^{(p)} \Phi \partial^{(s-p-2)} \overline{\mathrm{D}} \mathrm{D} \mathcal{K}_{\Phi}+\sum_{p=0}^{s-2} g_{p} \partial^{(p)} \bar{\Phi} \partial^{(s-p-2)} \overline{\mathrm{D}} \mathrm{D} \mathcal{K}_{\bar{\Phi}} \\
& +\sum_{p=0}^{s-2} \xi_{p} \partial^{(p)} \Phi \partial^{(s-p-2)} \mathrm{D} \overline{\mathrm{D}} \mathcal{K}_{\Phi} .
\end{aligned}
$$

\subsection{Conservation equation}

The above streamlined expressions do not include any trivial parts for the higher spin supercurrent and supertrace and are the ones we should use with the conservation equation. After a lengthy calculation and assuming once again that the Kähler potential is arbitrary, we obtain the following system of conditions:

1.) $i \delta+\kappa=0$,

2.) $\quad \alpha_{p}\left[\frac{s-p}{p}\right]-\beta_{p+1}\left[\frac{p+1}{s}\right]+i \xi_{p}=0, \quad p=0,1, \ldots, s-2$,

3.) $\quad \alpha_{s-2}\left[\frac{1}{s}\right]-\beta_{s-1}+\gamma_{s-1}\left[\frac{s+1}{s}\right]-\kappa+i \xi_{s-2}-i \zeta_{s-2}=0$,

4.) $\quad \alpha_{p-1}\left[\frac{s-p}{s}\right]-\beta_{p}\left[\frac{p+1}{s}\right]+\gamma_{p}\left[\frac{s+1}{s}\right]+i \xi_{p-1}-i \zeta_{p-1}-i \zeta_{p}=0$,

$$
p=1, \ldots, s-2,
$$



5.) $\quad \beta_{0}\left[\frac{1}{s}\right]-\gamma_{0}\left[\frac{s+1}{s}\right]+i \zeta_{0}=0$,
6.) $a_{p}\left[\frac{s+1}{s}\right]+i g_{p}=0, \quad p=0, \ldots, s-2$,
7.) $a_{p}\left[\frac{p+1}{s}\right]+i g_{p}=0, \quad p=0, \ldots, s-2$,
8.) $\quad c_{s-1}=0$,
9.) $c_{p}\left[\frac{p+1}{s}\right]-i g_{p}=0, \quad p=0, \ldots, s-2$,
10.) $\quad d=0$,
11.) $\quad c_{p}=0, \quad p=0, \ldots, s-1$,
12.) $\quad a_{p}+c_{p}=0, \quad p=0, \ldots, s-1$.

These uniquely fix all parameters except $\alpha_{p}, \beta_{p}$ and $\gamma_{p}$. Specifically we get the following solutions:

$$
\begin{aligned}
& d=0, \\
& a_{p}=c_{p}=0, \\
& \delta=\frac{i(-1)^{s-1}}{s}\left\{\sum_{i=0}^{s-2}(-1)^{i} \alpha_{i}-\sum_{i=0}^{s-1}(-1)^{i} \beta_{i}+(s+1) \sum_{i=0}^{s-1}(-1)^{i} \gamma_{i}\right\}, \\
& \kappa=\frac{(-1)^{s-1}}{s}\left\{\sum_{i=0}^{s-2}(-1)^{i} \alpha_{i}-\sum_{i=0}^{s-1}(-1)^{i} \beta_{i}+(s+1) \sum_{i=0}^{s-1}(-1)^{i} \gamma_{i}\right\} \text {, } \\
& \xi_{p}=\frac{i}{s}\left\{\alpha_{p}(s-p)-\beta_{p+1}(p+1)\right\} \text {, } \\
& \zeta_{p}=\frac{i(-1)^{p-1}}{s}\left\{\sum_{i=0}^{p-1}(-1)^{i} \alpha_{i}-\sum_{i=0}^{p}(-1)^{i} \alpha_{i}+(s+1) \sum_{i=0}^{p}(-1)^{i} \gamma_{i}\right\}, \quad p=0, \ldots, s-2, \\
& g_{p}=0, \\
& p=0, \ldots, s-1, \\
& p=0, \ldots, s-2, \\
& p=0, \ldots, s-2,
\end{aligned}
$$

and the conserved supercurrent multiplet we get is:

$$
\begin{aligned}
\mathcal{J}_{\alpha(s) \dot{\alpha}(s)}= & \delta \partial^{(s)} \Phi \mathcal{K}_{\Phi}+\sum_{p=0}^{s-2} \alpha_{p} \partial^{(p)} \mathrm{D} \Phi \partial^{(s-p-1)} \overline{\mathrm{D}} \mathcal{K}_{\Phi} \\
& +\sum_{p=0}^{s-1} \beta_{p} \partial^{(p)} \Phi \partial^{(s-p-1)} \mathrm{D} \overline{\mathrm{D}} \mathcal{K}_{\Phi}+\sum_{p=0}^{s-1} \gamma_{p} \partial^{(p)} \Phi \partial^{(s-p-1)} \overline{\mathrm{D}} \mathrm{D} \mathcal{K}_{\Phi} \\
\mathcal{T}_{\alpha(s-1) \dot{\alpha}(s-1)}= & \kappa \partial^{(s-1)} \Phi \mathcal{K}_{\Phi}+\sum_{p=0}^{s-2} \zeta_{p} \partial^{(p)} \Phi \partial^{(s-p-2)} \overline{\mathrm{D}} \mathrm{D} \mathcal{K}_{\Phi} \\
& +\sum_{p=0}^{s-2} \xi_{p} \partial^{(p)} \Phi \partial^{(s-p-2)} \mathrm{D} \overline{\mathrm{D}} \mathcal{K}_{\Phi}
\end{aligned}
$$

If we compare (5.18) with the corresponding expression for the $s=1$ case (4.9) we realize that there is a qualitative difference between the two supercurrents. In (4.9), the $\delta$ coef- 
ficient was not fixed, whereas for $s \geq 2$ the same coefficient is fixed and given by (5.17) which was the outcome of the third (3) condition in (5.16). The reason for that is exactly what was mentioned in (5.3). The $e \mathcal{K}$ term was independent in $s=1$, whereas it could be ignored in $s \geq 2$. However, if the Kähler potential was not arbitrary, but had the special property to remove the third condition in (5.16) by making the corresponding term vanish, then by accident we will be in the same situation as supergravity, where all coefficients in (5.18) are unconstrained. The corresponding term in the conservation equation that controls this is $\partial^{(s-1)} \Phi \overline{\mathrm{D}}^{2} \mathrm{D} \mathcal{K}_{\Phi}$. It will be interesting to consider potentials $\mathcal{K}$ that make this term vanish identically. Notice that the free theory $\mathcal{K}=\bar{\Phi} \Phi$ does that.

\subsection{Reality condition}

The last step in order to complete our construction is to search for real supercurrents of type (5.18). Using (4.11), we can write

$$
\begin{aligned}
\mathcal{J}_{\alpha(s) \dot{\alpha}(s)}= & \delta \partial^{(s)} \Phi \mathcal{K}_{\Phi}+\sum_{p=0}^{s-2} \alpha_{p} \partial^{(p)} \mathrm{D} \Phi \partial^{(s-p-1)}\left(\overline{\mathrm{D}} \bar{\Phi} \mathcal{K}_{\Phi \bar{\Phi}}\right) \\
& +\sum_{p=0}^{s-1} \beta_{p} \partial^{(p)} \Phi \partial^{(s-p-1)}\left(i \partial \bar{\Phi} \mathcal{K}_{\Phi \bar{\Phi}}+\mathrm{D} \Phi \overline{\mathrm{D}} \bar{\Phi} \mathcal{K}_{\Phi \Phi \bar{\Phi}}\right) \\
& +\sum_{p=0}^{s-1} \gamma_{p} \partial^{(p)} \Phi \partial^{(s-p-1)}\left(i \partial \Phi \mathcal{K}_{\Phi \Phi}-\mathrm{D} \Phi \overline{\mathrm{D}} \bar{\Phi} \mathcal{K}_{\Phi \Phi \bar{\Phi}}\right) .
\end{aligned}
$$

After distributing the derivatives and collecting similar terms, as we did in (4.12), we find the conditions imposed on coefficients $\alpha_{p}, \beta_{p}, \gamma_{p}$. To simplify this step we split $\mathcal{J}_{\alpha(s) \dot{\alpha}(s)}$ into two pieces

$$
\mathcal{J}_{\alpha(s) \dot{\alpha}(s)}=\mathcal{J}_{\alpha(s) \dot{\alpha}(s)}^{(\not)}+\mathcal{J}_{\alpha(s) \dot{\alpha}(s)}^{(\mathrm{D})}
$$

where $\mathcal{J}_{\alpha(s) \dot{\alpha}(s)}^{(D)}$ are the contributions to $\mathcal{J}_{\alpha(s) \dot{\alpha}(s)}$ without spinorial derivatives and $\mathcal{J}_{\alpha(s) \dot{\alpha}(s)}^{(\mathrm{D})}$ are the terms which include spinorial derivatives. This distinction is useful because terms from one piece can not contribute to the reality of the other, thus we can examine them separately. Therefore, if we ignore for the moment all the terms in (5.20) with spinorial derivatives we get:

$$
\begin{aligned}
\mathcal{J}_{\alpha(s) \dot{\alpha}(s)}^{(\not D)}= & \frac{\partial^{(s)} \Phi\left\{\delta \mathcal{K}_{\Phi}+i \gamma_{0} \Phi \mathcal{K}_{\Phi \Phi}\right\}+\partial^{(s)} \bar{\Phi}\left\{i \beta_{0} \Phi \mathcal{K}_{\Phi \bar{\Phi}}\right\}}{{ }^{s-1} \partial^{(p)} \Phi \partial^{(s-p)} \Phi\left\{i \gamma_{p} \mathcal{K}_{\Phi \Phi}\right\}} \\
& +\sum_{p=1}^{s-1} \partial^{(p)} \Phi \partial^{(s-p)} \bar{\Phi}\left\{i \beta_{p} \mathcal{K}_{\Phi \bar{\Phi}}\right\} \\
& +\sum_{p=1}^{s-1} \partial^{(s-p)} \Phi \partial^{(p)} \mathcal{K}_{\Phi \Phi}\left\{i \gamma_{0}\left(\begin{array}{c}
s-1 \\
p
\end{array}\right)\right\}+\sum_{p=1}^{s-1} \partial^{(s-p)} \bar{\Phi} \partial^{(p)} \mathcal{K}_{\Phi \bar{\Phi}}\left\{i \beta_{0}\left(\begin{array}{c}
s-1 \\
p
\end{array}\right)\right\}
\end{aligned}
$$




$$
\begin{aligned}
& +\sum_{p=1}^{s-2} \sum_{q=1}^{s-p-1} \partial^{(p)} \Phi \partial^{(s-p-q)} \Phi \partial^{(q)} \mathcal{K}_{\Phi \Phi}\left\{i \gamma_{p}\left(\begin{array}{c}
s-p-1 \\
q
\end{array}\right)\right\} \\
& +\sum_{p=1}^{s-2} \sum_{q=1}^{s-p-1} \partial^{(p)} \Phi \partial^{(s-p-q)} \bar{\Phi} \partial^{(q)} \mathcal{K}_{\Phi \Phi}\left\{i \beta_{p}\left(\begin{array}{c}
s-p-1 \\
q
\end{array}\right)\right\} .
\end{aligned}
$$

The reality of $(5.22)$ for an arbitrary $\mathcal{K}$ gives the following conditions: ${ }^{10}$

$$
\begin{array}{rlrl}
\delta & =\gamma_{0}=\beta_{0}=0, & & \\
\gamma_{p} & =0, & & p=1,2, \ldots, s-1, \\
\beta_{p} & =-\beta_{s-p}^{*}, & p=1,2, \ldots, s-1, \\
\beta_{p}\left(\begin{array}{c}
s-p-1 \\
q
\end{array}\right) & =-\beta_{s-p-q}^{*}\left(\begin{array}{c}
p+q-1 \\
q
\end{array}\right) . & &
\end{array}
$$

Doing the same for the reality of the terms with spinorial derivatives we get the constraints:

$$
\begin{array}{rlr}
\alpha_{0} & =0, & \\
\beta_{s-1} & =0, & \\
\beta_{p} & =0, & p=1,2, \ldots, s-2, \\
\alpha_{p} & =0, & p=1,2, \ldots, s-2 .
\end{array}
$$

This is because by using $\beta_{0}=\gamma_{p}=0$, the terms in $\mathcal{J}_{\alpha(s) \dot{\alpha}(s)}^{(\mathrm{D})}$ can be written in the following manner:

$$
\begin{aligned}
\mathcal{J}_{\alpha(s) \dot{\alpha}(s)}^{(\mathrm{D})}= & \frac{\mathrm{D} \Phi \partial^{(s-1)} \overline{\mathrm{D}} \bar{\Phi}\left\{\alpha_{0} \mathcal{K}_{\Phi \bar{\Phi}\}}\right\}}{}+\sum_{p=1}^{s-2} \partial^{(p)} \mathrm{D} \Phi \partial^{(s-p-1)} \overline{\mathrm{D}} \bar{\Phi}\left\{\alpha_{p} \mathcal{K}_{\Phi \bar{\Phi}}\right\} \\
& +\sum_{p=1}^{s-2} \mathrm{D} \Phi \partial^{(s-p-1)} \overline{\mathrm{D}} \bar{\Phi} \partial^{(p)} \mathcal{K}_{\Phi \bar{\Phi}}\left\{\alpha_{0}\left(\begin{array}{c}
s-1 \\
p
\end{array}\right)\right\} \\
& +\sum_{p=1}^{s-2} \partial^{(p)} \Phi \mathrm{D} \Phi \partial^{(s-p-1)} \overline{\mathrm{D}} \bar{\Phi}\left\{\beta_{p} \mathcal{K}_{\Phi \Phi \bar{\Phi}}\right\} \\
& +\sum_{p=1}^{s-2} \sum_{q=1}^{s-p-2} \partial^{(p)} \Phi \mathrm{D} \Phi \partial^{(s-p-q-1)} \overline{\mathrm{D}} \bar{\Phi} \partial^{(q)} \mathcal{K}_{\Phi \Phi \bar{\Phi}}\left\{\beta_{p}\left(\begin{array}{c}
s-p-1 \\
q
\end{array}\right)\right\} \\
& +\sum_{p=1}^{s-2} \partial^{(s-p-1)} \mathrm{D} \Phi \overline{\mathrm{D}} \bar{\Phi} \partial^{(p)} \mathcal{K}_{\Phi \bar{\Phi}}\left\{\alpha_{s-p-1}\right\} \\
& +\sum_{p=1}^{s-2} \partial^{(p)} \Phi \partial^{(s-p-1)} \mathrm{D} \Phi \overline{\mathrm{D}} \bar{\Phi}\left\{\beta_{p} \mathcal{K}_{\Phi \Phi \bar{\Phi}}\right\}
\end{aligned}
$$

\footnotetext{
${ }^{10}$ We have underlined the relevant terms in order for the reader to track the origin of these conditions.
} 


$$
\begin{aligned}
& +\sum_{p=1}^{s-2} \sum_{q=1}^{s-p-2} \partial^{(p)} \Phi \partial^{(s-p-q-1)} \mathrm{D} \Phi \overline{\mathrm{D}} \bar{\Phi} \partial^{(q)} \mathcal{K}_{\Phi \Phi \bar{\Phi}}\left\{\beta_{p}\left(\begin{array}{c}
s-p-1 \\
q
\end{array}\right)\right\} \\
& +\mathrm{D} \Phi \overline{\mathrm{D}} \bar{\Phi} \partial^{(s-1)} \mathcal{K}_{\Phi \bar{\Phi}}\left\{\alpha_{0}\right\}+\overline{\partial^{(s-1)} \Phi \mathrm{D} \Phi \overline{\mathrm{D}} \bar{\Phi}\left\{\beta_{s-1} \mathcal{K}_{\Phi \Phi \bar{\Phi}\}}\right.} \\
& +\sum_{p=1}^{s-2} \partial^{(p)} \Phi \mathrm{D} \Phi \overline{\mathrm{D}} \bar{\Phi} \partial^{(s-p-1)} \mathcal{K}_{\Phi \Phi \bar{\Phi}}\left\{\beta_{p}\right\} \\
& +\sum_{p=1}^{s-2} \sum_{q=1}^{s-p-2} \partial^{(p)} \mathrm{D} \Phi \partial^{(s-p-q-1)} \overline{\mathrm{D}} \bar{\Phi} \partial^{(q)} \mathcal{K}_{\Phi \bar{\Phi}}\left\{\alpha_{p}\left(\begin{array}{c}
s-p-1 \\
q
\end{array}\right)\right\} \\
& +\sum_{p=1}^{s-2} \sum_{q=1}^{s-p-2} \partial^{(p)} \Phi \partial^{(q)} \mathrm{D} \Phi \partial^{(s-p-q-1)} \overline{\mathrm{D}} \bar{\Phi}\left\{\beta_{p}\left(\begin{array}{c}
s-p-1 \\
q
\end{array}\right) \mathcal{K}_{\Phi \Phi \bar{\Phi}}\right\} \\
& +\sum_{p=1}^{s-2} \sum_{q=1}^{s-p-2} s \sum_{r=1}^{s-p-q-2} \partial^{(p)} \Phi \partial^{(r)} \mathrm{D} \Phi \partial^{(s-p-q-r-1)} \overline{\mathrm{D}} \bar{\Phi} \partial^{(q)} \\
& \times \mathcal{K}_{\Phi \Phi \bar{\Phi}}\left\{\beta_{p}\left(\begin{array}{c}
s-p-1 \\
q
\end{array}\right)\left(\begin{array}{c}
s-p-q-1 \\
r
\end{array}\right)\right\} .
\end{aligned}
$$

The conclusion is that, in order to get a real, higher spin supercurrent all coefficients $\alpha_{p}, \beta_{p}, \gamma_{p}$ must vanish. Therefore, there is no non-trivial solution for arbitrary Kähler potential $\mathcal{K}$.

However, an interesting question one can ask is whether there is a special Kähler potential $\mathcal{K}^{s}$ such that we can construct non-trivial real, higher spin supercurrents. After all, we have seen this behavior in the coupling with the vector supermultiplet, where the Kähler potential must have a U(1) symmetry. Therefore, one can imagine that if the special property of $\mathcal{K}^{s}$ is a realization of higher spin symmetry then maybe the higher spin supercurrent exist. Going back to (5.25) and examining the terms responsible for the vanishing of $\beta_{p}$ and $\alpha_{p}$ we find that a necessary condition for $\mathcal{K}^{s}$ is:

$$
\mathcal{K}_{\Phi \Phi \bar{\Phi}}^{s}=0 \text {. }
$$

This is equivalent to $\mathcal{K}_{\Phi \bar{\Phi}}^{s}=$ constant which holds true only for the free theory $\mathcal{K}^{s}=$ $\bar{\Phi} \Phi$. Furthermore, this condition is consistent because for the free theory the term $\partial^{(s-1)} \Phi \overline{\mathrm{D}}^{2} \mathrm{D} \mathcal{K}_{\Phi}$ leading to the fixing of $\delta$ in (5.17) vanishes, hence there is no incompatibility between the non-trivial values of the parameters and conservation equation. Additionally, the quantity $Z_{\alpha(s-1) \dot{\alpha}(s-1)}(5.10)$ becomes identically zero for the free theory. So the parameters we removed, such as $\alpha_{s-1}$ become relevant now. All these accidents take place only if (5.26) is true and for that case we recover the results of $[56,57]$.

\section{Turn on the chiral superpotential $\mathcal{W}$}

In this section, we turn back on the chiral superpotential $\mathcal{W}(\Phi)$ in order to study its contribution to the higher spin supercurrent multiplet, when that is possible. We must keep in mind that $\mathcal{W}$ is a chiral superfield $\left(\overline{\mathrm{D}}_{\dot{\alpha}} \mathcal{W}=0\right)$ and the on-shell equation of motion now becomes $\overline{\mathrm{D}}^{2} \mathcal{K}_{\Phi}=\mathcal{W}_{\Phi}$. 


\subsection{For vector supermultiplet}

We start with supercurrent (3.8) and we modify it in order to include the chiral superpotential information while preserving its reality:

$$
\mathcal{J}=\Phi \mathcal{K}_{\Phi}+c \Lambda \mathcal{W}_{\Phi}+c^{*} \bar{\Lambda} \overline{\mathcal{W}}_{\bar{\Phi}}
$$

where $\Lambda$ is the prepotential of the chiral superfield, $\Phi=\overline{\mathrm{D}}^{2} \Lambda$. It is straight forward to prove that the conservation equation of $\mathcal{J}$

$$
\overline{\mathrm{D}}^{2} \mathcal{J}=(1+c) \Phi \mathcal{W}_{\Phi}+c^{*} \overline{\mathrm{D}}^{2} \bar{\Lambda} \overline{\mathcal{W}}_{\bar{\Phi}}+c^{*} \overline{\mathrm{D}}^{\dot{\beta}} \bar{\Lambda} \overline{\mathrm{D}}_{\dot{\beta}} \overline{\mathcal{W}}_{\bar{\Phi}}+c^{*} \bar{\Lambda} \overline{\mathrm{D}}^{2} \overline{\mathcal{W}}_{\bar{\Phi}}=0
$$

can not be satisfied for any value of $c$. Hence, the conclusion is that in the presence of any chiral superpotential $\mathcal{W}$, there can be no conserved supercurrent no matter what the Kähler potential is, even for the free theory. This can be understood as the fact that the presence of $\mathcal{W}$ breaks the global $\mathrm{U}(1)$ symmetry of section 3 .

\subsection{For supergravity}

For the supercurrent multiplet (4.16), (4.17) that generates interactions with the supergravity supermultiplet we consider the following modification terms

$$
\begin{aligned}
\mathcal{J}_{\alpha \dot{\alpha}}= & \mathrm{D}_{\alpha} \Phi \overline{\mathrm{D}}_{\dot{\alpha}} \mathcal{K}_{\Phi}+c_{1} \mathrm{D}_{\alpha} \overline{\mathrm{D}}_{\dot{\alpha}} \Lambda \mathcal{F}+c_{2} \overline{\mathrm{D}}_{\dot{\alpha}} \Lambda \mathrm{D}_{\alpha} \mathcal{F}+c_{3} \Lambda \overline{\mathrm{D}}_{\dot{\alpha}} \mathrm{D}_{\alpha} \mathcal{F}+c_{4} \overline{\mathrm{D}}_{\dot{\alpha}} \mathrm{D}_{\alpha} \Lambda \mathcal{F} \\
& -c_{1}^{*} \overline{\mathrm{D}}_{\dot{\alpha}} \mathrm{D}_{\alpha} \bar{\Lambda} \overline{\mathcal{F}}-c_{2}^{*} \mathrm{D}_{\alpha} \bar{\Lambda} \overline{\mathrm{D}}_{\dot{\alpha}} \overline{\mathcal{F}}-c_{3}^{*} \bar{\Lambda} \mathrm{D}_{\alpha} \overline{\mathrm{D}}_{\dot{\alpha}} \overline{\mathcal{F}}-c_{4}^{*} \mathrm{D}_{\alpha} \overline{\mathrm{D}}_{\dot{\alpha}} \bar{\Lambda} \overline{\mathcal{F}} \\
\mathcal{T}= & -\mathcal{K}+d_{1} \Lambda \mathcal{F}+d_{2} \bar{\Lambda} \overline{\mathcal{F}}
\end{aligned}
$$

In the above terms, $\mathcal{F}=\mathcal{F}(\Phi)$ is a chiral superfield and a holomorphic function of $\Phi$ defined as

$$
\mathcal{W}(\Phi)=\Phi \mathcal{F}(\Phi)
$$

This definition holds for any chiral superpotential because its Taylor expansion does not include the constant term due to (2.7). Additionally, it relates $\mathcal{W}_{\Phi}$ which appears in the equation of motion with $\mathcal{F}$ in the following manner:

$$
\mathcal{W}_{\Phi}=\mathcal{F}+\Phi \mathcal{F}_{\Phi}
$$

Imposing the conservation equation (4.1) we get a non-trivial solution

$$
\begin{array}{ll}
c_{1}=-1, & d_{1}=1, \\
c_{2}=1, & d_{2}=2, \\
c_{3}=0, & c_{4}=0 .
\end{array}
$$

Thus, the supercurrent multiplet (4.16), (4.17) can be generalized to include an arbitrary chiral superpotential

$$
\begin{aligned}
\mathcal{J}_{\alpha \dot{\alpha}} & =\mathrm{D}_{\alpha} \Phi \overline{\mathrm{D}}_{\dot{\alpha}} \mathcal{K}_{\Phi}-\mathrm{D}_{\alpha} \overline{\mathrm{D}}_{\dot{\alpha}}(\Lambda \mathcal{F})+\overline{\mathrm{D}}_{\dot{\alpha}} \mathrm{D}_{\alpha}(\bar{\Lambda} \overline{\mathcal{F}}), \\
\mathcal{T} & =-\mathcal{K}+\Lambda \mathcal{F}+2 \bar{\Lambda} \overline{\mathcal{F}} .
\end{aligned}
$$




\subsection{For higher spin supermultiplet}

In section 5 we showed that the construction of the higher spin supercurrent multiplet is possible only for the free theory, $\mathcal{K}=\bar{\Phi} \Phi$. For that case [56, 57], there is the minimal multiplet

$$
\begin{aligned}
\mathcal{J}_{\alpha(s) \dot{\alpha}(s)}^{\text {free }}= & c(-i)^{s} \sum_{p=0}^{s}(-1)^{p}\left(\begin{array}{l}
s \\
p
\end{array}\right)^{2} \partial^{(p)} \Phi \partial^{(s-p)} \bar{\Phi} \\
& +i c(-i)^{s} \sum_{p=0}^{s-1}(-1)^{p}\left(\begin{array}{c}
s \\
p
\end{array}\right)^{2} \frac{s-p}{p+1} \partial^{(p)} \mathrm{D} \Phi \partial^{(s-p-1)} \overline{\mathrm{D}} \bar{\Phi}, \\
\mathcal{T}_{\alpha(s-1) \dot{\alpha}(s-1)}^{\text {free }}= & 0,
\end{aligned}
$$

where $c$ is a real proportionality constant (it may depend on the value of $s$ ). Hence, the consideration of contributions due to the presence of a chiral superpotential $\mathcal{W}$ must take place in the same configuration. The most general ansatz for the $\mathcal{W}$ generated terms are:

$$
\begin{aligned}
\mathcal{J}_{\alpha(s) \dot{\alpha}(s)}^{\mathcal{W}}= & \sum_{p=0}^{s-1} \gamma_{p} \partial^{(p)} \mathrm{D} \overline{\mathrm{D}} \Lambda \partial^{(s-p-1)} \mathcal{W}_{\Phi}+\sum_{p=0}^{s-1} \delta_{p} \partial^{(p)} \overline{\mathrm{D}} \Lambda \partial^{(s-p-1)} \mathrm{D} \mathcal{W}_{\Phi} \\
& -\sum_{p=0}^{s-1} \gamma_{p}^{*} \partial^{(p)} \overline{\mathrm{D}} \mathrm{D} \bar{\Lambda} \partial^{(s-p-1)} \overline{\mathcal{W}}_{\bar{\Phi}}-\sum_{p=0}^{s-1} \delta_{p}^{*} \partial^{(p)} \mathrm{D} \bar{\Lambda} \partial^{(s-p-1)} \overline{\mathrm{D}}_{\overline{\mathcal{\Phi}}}, \\
\mathcal{T}_{\alpha(s-1) \dot{\alpha}(s-1)}^{\mathcal{W}} & \sum_{p=0}^{s-1} \zeta_{p} \partial^{(p)} \Lambda \partial^{(s-p-1)} \mathcal{W}_{\Phi}+\sum_{p=0}^{s-2} \sigma_{p} \partial^{(p)} \overline{\mathrm{D}} \mathrm{D} \Lambda \partial^{(s-p-2)} \mathcal{W}_{\Phi} \\
& +\sum_{p=0}^{s-1} \xi_{p} \partial^{(p)} \bar{\Lambda} \partial^{(s-p-1)} \overline{\mathcal{W}}_{\bar{\Phi}},
\end{aligned}
$$

and the conservation equation they must satisfy is

$$
\overline{\mathrm{D}}^{\dot{\alpha}_{s}} \mathcal{J}_{\alpha(s) \dot{\alpha}(s)}^{\text {free }}+\overline{\mathrm{D}}^{\dot{\alpha}_{s}} \mathcal{J}_{\alpha(s) \dot{\alpha}(s)}^{\mathcal{W}}-\frac{1}{s !} \overline{\mathrm{D}}^{2} \mathrm{D}_{\left(\alpha_{s}\right.} \mathcal{T}_{\alpha(s-1)) \dot{\alpha}(s-1)}^{\mathcal{W}}=0 .
$$

Substituting (6.11), (6.12) in (6.13), the cancellation of the $\Lambda$ and $\bar{\Lambda}$ dependent terms gives:

$$
\begin{array}{ll}
\delta_{p}=-\gamma_{p}, & p=0,1, \ldots, s-1, \\
\xi_{p}=-\frac{s+1}{s} \gamma_{p}^{*}, & p=0,1, \ldots, s-1, \\
\zeta_{0}=-\frac{1}{s} \gamma_{0}, & p=1, \ldots, s-1, \\
\zeta_{p}=-\frac{p+1}{s} \gamma_{p}+\frac{s-p}{s} \gamma_{p-1}, & \\
i \sigma_{0}=\frac{1}{s} \gamma_{1}-\frac{s-1}{s} \gamma_{0}, & \\
i \sigma_{s-2}=\frac{s-1}{s} \gamma_{s-1}-\frac{1}{s} \gamma_{s-2}, & \\
i \sigma_{p}+i \sigma_{p-1}=\frac{p+1}{s} \gamma_{p+1}-\frac{s-2 p-1}{s} \gamma_{p}-\frac{s-p}{s} \gamma_{p-1}, & p=0,1, \ldots, s-1 .
\end{array}
$$


These are exactly the conditions found in [57]. The cancellation of the $\Phi$ dependent terms gives:

$$
\begin{aligned}
0= & \left\{c(i)^{s+1}(s+1)-\gamma_{s-1}\right\} \partial^{(s-1)} \mathrm{D} \Phi \mathcal{W}_{\Phi}+\left\{c(-i)^{s-1}-\gamma_{0}\right\} \Phi \partial^{(s-1)} \mathrm{D} \mathcal{W}_{\Phi} \\
& +\sum_{p=0}^{s-2}\left\{c(-i)^{s+1}(-1)^{p} \frac{s+1}{s}\left(\begin{array}{c}
s \\
p
\end{array}\right)^{2} \frac{s-p}{p+1}-\frac{p+1}{s}\left(\gamma_{p+1}+\gamma_{p}\right)\right\} \partial^{(p)} \mathrm{D} \Phi \partial^{(s-p-1)} \mathcal{W}_{\Phi} \\
& +\sum_{p=0}^{s-2} \frac{s-p-1}{p+1}\left\{c(-i)^{s+1}(-1)^{p} \frac{s+1}{s}\left(\begin{array}{c}
s \\
p
\end{array}\right)^{2} \frac{s-p}{p+1}-\frac{p+1}{s}\left(\gamma_{p+1}+\gamma_{p}\right)\right\} \\
& \times \partial^{(p+1)} \Phi \partial^{(s-p-2)} \mathrm{D} \mathcal{W}_{\Phi} .
\end{aligned}
$$

Therefore, for an arbitrary chiral superpotential we get the constraints

$$
\begin{aligned}
\gamma_{s-1} & =c(i)^{s+1}(s+1), \\
\gamma_{0} & =c(-i)^{s-1}, \\
\gamma_{p+1}+\gamma_{p} & =c(-i)^{s+1}(-1)^{p}(s+1)\left(\begin{array}{l}
s \\
p
\end{array}\right)^{2} \frac{s-p}{(p+1)^{2}}, \quad p=0,1, \ldots, s-2 .
\end{aligned}
$$

One can easily check that the above equations are not consistent with each other. For example, starting with $\gamma_{0}$ and using the recursive equation $(6.16 \mathrm{c})$ we reach to a different value of $\gamma_{s-1}$ than the one required. The conclusion is that for an arbitrary chiral superpotential $\mathcal{W}$, there is no higher spin supercurrent multiplet.

Nevertheless, careful examination of (6.15) reveals two exceptions exist for some special superpotential $\mathcal{W}^{s}$. The first one is for

$$
\mathcal{W}^{s} \sim \Phi^{2} .
$$

In this case, the various terms of (6.15) are no longer independent and they can be combined, resulting to a different set of constraints for the $\gamma_{p}$ parameters. Of course, (6.17) corresponds to the mass term of the chiral superfield and equation (6.15) will lead to the analysis of [57] were the mass term contributions to the higher spin supercurrent and supertrace were calculated. The main result was that only the odd values of $s(s=2 l+1)$ will lead to consistent, interactions. Additionally, a second exception exist and corresponds to a linear superpotential

$$
\mathcal{W}^{s}=f \Phi
$$

In that case all the terms of (6.15) with derivatives acting on $\mathcal{W}_{\Phi}$ will vanish and we get only condition (6.16a). The corresponding supercurrent multiplet is:

$$
\begin{aligned}
\mathcal{J}_{\alpha(s) \dot{\alpha}(s)}= & \mathcal{J}_{\alpha(s) \dot{\alpha}(s)}^{\text {free }}+f c(s+1)(i)^{s+1} \partial^{(s-1)} \mathrm{D} \overline{\mathrm{D}} \Lambda-\bar{f} c(s+1)(-i)^{s+1} \partial^{(s-1)} \overline{\mathrm{D}} \bar{\Lambda}, \\
\mathcal{T}_{\alpha(s-1) \dot{\alpha}(s-1)}= & -f c(s+1)(i)^{s+1} \partial^{(s-1)} \Lambda+f c \frac{s^{2}-1}{s}(i)^{s} \partial^{(s-2)} \overline{\mathrm{D}} \mathrm{D} \Lambda \\
& -\bar{f} c \frac{(s+1)^{2}}{s}(-i)^{s+1} \partial^{(s-1)} \bar{\Lambda} .
\end{aligned}
$$

and it exists for all values of $s$. 


\section{Summary and discussion}

In this paper, we have investigated the interactions of higher spin gauge fields with matter theory fields independently from the ability to have a properly define $\mathcal{S}$-matrix. We considered an interacting matter theory described by a chiral superfield $\Phi$ with its dynamics been described an arbitrary Kähler potential $\mathcal{K}(\Phi, \bar{\Phi})$ and a chiral superpotential $\mathcal{W}(\Phi)$. The arbitrariness of both $\mathcal{K}$ and $\mathcal{W}$ allows us to parametrize some very complicated, strong interactions which can disrupt the conventional definition of free in and out states and thus evading the consequences of the Coleman-Mandula theorem. We have also assumed that the first order interactions of the matter theory with higher spin supermultiplets of type $(s+1, s+1 / 2)$, if they exist, are generated by a higher spin supercurrent multiplet $\left(\mathcal{J}_{\alpha(s) \dot{\alpha}(s)}, T_{\alpha(s-1) \dot{\alpha}(s-1)}\right)$ defined by a real higher spin supercurrent $\mathcal{J}_{\alpha(s) \dot{\alpha}(s)}$ and a higher spin supertrace $\mathcal{T}_{\alpha(s-1) \dot{\alpha}(s-1)}$

$$
S_{1} \sim \int d^{8} z\left\{H^{\alpha(s) \dot{\alpha}(s)} \mathcal{J}_{\alpha(s) \dot{\alpha}(s)}+\chi^{\alpha(s) \dot{\alpha}(s-1)} \mathrm{D}_{\alpha_{s}} \mathcal{T}_{\alpha(s-1) \dot{\alpha}(s-1)}+\bar{\chi}^{\alpha(s-1) \dot{\alpha}(s)} \overline{\mathrm{D}}_{\dot{\alpha}_{s}} \overline{\mathcal{T}}_{\alpha(s-1) \dot{\alpha}(s-1)}\right\}
$$

and on-shell satisfy the conservation equation

$$
\overline{\mathrm{D}}^{\dot{\alpha}_{s}} \mathcal{J}_{\alpha(s) \dot{\alpha}(s)}=\frac{1}{s !} \overline{\mathrm{D}}^{2} \mathrm{D}_{\left(\alpha_{s}\right.} \mathcal{T}_{\alpha(s-1)) \dot{\alpha}(s-1)} .
$$

The results we find are:

(i) For $s=0$ (vector supermultiplet) there are no interactions unless the Kähler potential can be written as a function of the product $\bar{\Phi} \Phi[\mathcal{K}=\mathcal{K}(\bar{\Phi} \Phi)]$ and the chiral superpotential vanishes $[\mathcal{W}(\Phi)=0]$. For these cases, the supercurrent is

$$
\mathcal{J}=\Phi \mathcal{K}_{\Phi}
$$

and satisfies the conservation equation $\overline{\mathrm{D}}^{2} \mathcal{J}=0$. The constraints in $\mathcal{K}$ and $\mathcal{W}$ can be understood as the global U(1) symmetry requirement for the gauging procedure which will generate the interactions with the vector supermultiplet.

(ii) For $s=1$ (non-minimal supergravity supermultiplet) the expectation is that we should always be able to find interactions. This was verified by our approach because for any $\mathcal{K}$ and $\mathcal{W}$ we can construct the following supercurrent multiplet

$$
\begin{aligned}
\mathcal{J}_{\alpha \dot{\alpha}} & =\mathrm{D}_{\alpha} \Phi \overline{\mathrm{D}}_{\dot{\alpha}} \mathcal{K}_{\Phi}-\mathrm{D}_{\alpha} \overline{\mathrm{D}}_{\dot{\alpha}}(\Lambda \mathcal{F})+\overline{\mathrm{D}}_{\dot{\alpha}} \mathrm{D}_{\alpha}(\bar{\Lambda} \overline{\mathcal{F}}) \\
\mathcal{T} & =-\mathcal{K}+\Lambda \mathcal{F}+2 \bar{\Lambda} \overline{\mathcal{F}}
\end{aligned}
$$

where $\Phi=\overline{\mathrm{D}}^{2} \Lambda$ and $\mathcal{W}=\Phi \mathcal{F}$. Furthermore, this result is consistent with the results of $[63]$.

(iii) For $s \geq 2$ (higher spin supermultiplets) there is a severe constraining of both $\mathcal{K}$ and $\mathcal{W}$. For almost any Kähler potential and chiral superpotential there are no interactions with higher spin supermultiplets. In other words, one can not construct 
a non-trivial higher spin supercurrent $\mathcal{J}_{\alpha(s) \dot{\alpha}(s)}$ and supertrace $\mathcal{T}_{\alpha(s-1) \dot{\alpha}(s-1)}$. However there are three exceptions:

1.) $\mathcal{K}=\bar{\Phi} \Phi, \quad \mathcal{W}=0: \quad$ free, massless, chiral superfield

2.) $\mathcal{K}=\bar{\Phi} \Phi, \quad \mathcal{W}=f \Phi: \quad$ free, chiral superfield with linear superpotential

3.) $\mathcal{K}=\bar{\Phi} \Phi, \quad \mathcal{W}=m \Phi^{2}:$ free, massive, chiral superfield

These exceptions are consistent with various known no-go theorems such as the Coleman-Mandula theorem [4] and its extensions to supersymmetry [5, 6]. For exceptions (1) and (3) the corresponding supercurrent multiplet have been constructed in [56-58]. To this list we add the supercurrent multiplet for exception (2)

$$
\begin{aligned}
\mathcal{J}_{\alpha(s) \dot{\alpha}(s)}= & \mathcal{J}_{\alpha(s) \dot{\alpha}(s)}^{\text {free }}+f c(s+1)(i)^{s+1} \partial^{(s-1)} \mathrm{D} \overline{\mathrm{D}} \Lambda-\bar{f} c(s+1)(-i)^{s+1} \partial^{(s-1)} \overline{\mathrm{D}} \bar{\Lambda}, \\
\mathcal{T}_{\alpha(s-1) \dot{\alpha}(s-1)=} & -f c(s+1)(i)^{s+1} \partial^{(s-1)} \Lambda+f c \frac{s^{2}-1}{s}(i)^{s} \partial^{(s-2)} \overline{\mathrm{D}} \mathrm{D} \Lambda \\
& -\bar{f} c \frac{(s+1)^{2}}{s}(-i)^{s+1} \partial^{(s-1)} \bar{\Lambda} .
\end{aligned}
$$

\section{Acknowledgments}

The research of I. L. B. was supported in parts by Russian Ministry of Education and Science, project No. 3.1386.2017. He is also grateful to RFBR grant, project No. 18-0200153 for partial support. The research of S. J. G. is supported by the endowment of the Ford Foundation Professorship of Physics at Brown University. The work of K. K. was supported by the grant P201/12/G028 of the Grant agency of Czech Republic. K. K. is thankful to Rikard von Unge and Linus Wulff for useful discussions.

Open Access. This article is distributed under the terms of the Creative Commons Attribution License (CC-BY 4.0), which permits any use, distribution and reproduction in any medium, provided the original author(s) and source are credited.

\section{References}

[1] S. Weinberg, Photons and gravitons in $S$ matrix theory: derivation of charge conservation and equality of gravitational and inertial mass, Phys. Rev. 135 (1964) B1049 [INSPIRE].

[2] S. Weinberg, The quantum theory of fields. Volume I: foundations, section 13.1, Cambridge University Press, Cambridge U.K., (1995) [INSPIRE].

[3] M.T. Grisaru, H.N. Pendleton and P. van Nieuwenhuizen, Supergravity and the S matrix, Phys. Rev. D 15 (1977) 996 [INSPIRE].

[4] S.R. Coleman and J. Mandula, All possible symmetries of the $S$ matrix, Phys. Rev. 159 (1967) 1251 [INSPIRE].

[5] R. Haag, J.T. Lopuszanski and M. Sohnius, All possible generators of supersymmetries of the S matrix, Nucl. Phys. B 88 (1975) 257 [INSPIRE].

[6] S. Weinberg, The quantum theory of fields. Volume III: supersymmetry, chapter 24, Cambridge University Press, Cambridge U.K., (2000) [INSPIRE]. 
[7] C. Aragone and S. Deser, Consistency problems of hypergravity, Phys. Lett. B 86 (1979) 161 [INSPIRE].

[8] F.A. Berends, J.W. van Holten, B. de Wit and P. van Nieuwenhuizen, On spin 5/2 gauge fields, J. Phys. A 13 (1980) 1643 [InSPIRE].

[9] C. Aragone and H. La Roche, Massless second order tetradic spin 3 fields and higher helicity bosons, Nuovo Cim. A 72 (1982) 149 [INSPIRE].

[10] S. Deser and Z. Yang, Inconsistency of spin 4-spin 2 gauge field couplings, Class. Quant. Grav. 7 (1990) 1491 [InSPIRE].

[11] M. Porrati, Universal limits on massless high-spin particles, Phys. Rev. D 78 (2008) 065016 [arXiv:0804.4672] [INSPIRE].

[12] M. Taronna, On the non-local obstruction to interacting higher spins in flat space, JHEP 05 (2017) 026 [arXiv: 1701.05772] [INSPIRE].

[13] R. Roiban and A.A. Tseytlin, On four-point interactions in massless higher spin theory in flat space, JHEP 04 (2017) 139 [arXiv: 1701.05773] [INSPIRE].

[14] C. Sleight and M. Taronna, Higher spin gauge theories and bulk locality: a no-go result, arXiv: 1704.07859 [INSPIRE].

[15] N. Boulanger and S. Leclercq, Consistent couplings between spin-2 and spin-3 massless fields, JHEP 11 (2006) 034 [hep-th/0609221] [INSPIRE].

[16] N. Boulanger, S. Leclercq and P. Sundell, On the uniqueness of minimal coupling in higher-spin gauge theory, JHEP 08 (2008) 056 [arXiv:0805.2764] [INSPIRE].

[17] X. Bekaert, N. Boulanger and P. Sundell, How higher-spin gravity surpasses the spin two barrier: no-go theorems versus yes-go examples, Rev. Mod. Phys. 84 (2012) 987 [arXiv:1007.0435] [INSPIRE].

[18] M. Porrati, Old and new no go theorems on interacting massless particles in flat space, in Proceedings, $17^{\text {th }}$ International Seminar on High Energy Physics (Quarks 2012), Yaroslavl Russia, 4-7 June 2012 [arXiv:1209.4876] [INSPIRE].

[19] A.K.H. Bengtsson, I. Bengtsson and L. Brink, Cubic interaction terms for arbitrary spin, Nucl. Phys. B 227 (1983) 31 [InSPIRE].

[20] A.K.H. Bengtsson, I. Bengtsson and L. Brink, Cubic interaction terms for arbitrarily extended supermultiplets, Nucl. Phys. B 227 (1983) 41 [INSPIRE].

[21] A.K.H. Bengtsson, I. Bengtsson and N. Linden, Interacting higher spin gauge fields on the light front, Class. Quant. Grav. 4 (1987) 1333 [INSPIRE].

[22] E.S. Fradkin and R.R. Metsaev, A cubic interaction of totally symmetric massless representations of the Lorentz group in arbitrary dimensions, Class. Quant. Grav. 8 (1991) L89 [inSPIRE].

[23] R.R. Metsaev, Note on the cubic interaction of massless representations of the Poincaré group in D $=5$ space-time, Class. Quant. Grav. 10 (1993) L39 [INSPIRE].

[24] R.R. Metsaev, Cubic interaction vertices of totally symmetric and mixed symmetry massless representations of the Poincaré group in D=6 space-time, Phys. Lett. B 309 (1993) 39 [INSPIRE].

[25] E.S. Fradkin and R.R. Metsaev, Cubic scattering amplitudes for all massless representations of the Poincaré group in any space-time dimension, Phys. Rev. D 52 (1995) 4660 [INSPIRE]. 
[26] R.R. Metsaev, Cubic interaction vertices for fermionic and bosonic arbitrary spin fields, Nucl. Phys. B 859 (2012) 13 [arXiv:0712.3526] [INSPIRE].

[27] R.R. Metsaev, Generating function for cubic interaction vertices of higher spin fields in any dimension, Mod. Phys. Lett. A 8 (1993) 2413 [INSPIRE].

[28] R.R. Metsaev, Cubic interaction vertices of massive and massless higher spin fields, Nucl. Phys. B 759 (2006) 147 [hep-th/0512342] [inSPIRE].

[29] F.A. Berends, G.J.H. Burgers and H. van Dam, On the theoretical problems in constructing interactions involving higher spin massless particles, Nucl. Phys. B 260 (1985) 295 [INSPIRE].

[30] F.A. Berends, G.J.H. Burgers and H. Van Dam, On spin three selfinteractions, Z. Phys. C 24 (1984) 247 [INSPIRE].

[31] F.A. Berends, G.J.H. Burgers and H. van Dam, Explicit construction of conserved currents for massless fields of arbitrary spin, Nucl. Phys. B 271 (1986) 429 [InSPIRE].

[32] R. Manvelyan, K. Mkrtchyan and W. Rühl, Off-shell construction of some trilinear higher spin gauge field interactions, Nucl. Phys. B 826 (2010) 1 [arXiv:0903.0243] [INSPIRE].

[33] O.A. Gelfond, E.D. Skvortsov and M.A. Vasiliev, Higher spin conformal currents in Minkowski space, Theor. Math. Phys. 154 (2008) 294 [hep-th/0601106] [INSPIRE].

[34] X. Bekaert, N. Boulanger and S. Leclercq, Strong obstruction of the Berends-Burgers-van Dam spin-3 vertex, J. Phys. A 43 (2010) 185401 [arXiv:1002.0289] [InSPIRE].

[35] R. Manvelyan, K. Mkrtchyan and W. Ruehl, Direct construction of a cubic selfinteraction for higher spin gauge fields, Nucl. Phys. B 844 (2011) 348 [arXiv:1002.1358] [INSPIRE].

[36] R. Manvelyan, K. Mkrtchyan and W. Rühl, General trilinear interaction for arbitrary even higher spin gauge fields, Nucl. Phys. B 836 (2010) 204 [arXiv:1003.2877] [INSPIRE].

[37] I.G. Koh and S. Ouvry, Interacting gauge fields of any spin and symmetry, Phys. Lett. B 179 (1986) 115 [Erratum ibid. B 183 (1987) 434] [INSPIRE].

[38] A.K.H. Bengtsson, BRST approach to interacting higher spin gauge fields, Class. Quant. Grav. 5 (1988) 437 [InSPIRE].

[39] L. Cappiello, M. Knecht, S. Ouvry and J. Stern, BRST construction of interacting gauge theories of higher spin fields, Annals Phys. 193 (1989) 10 [INSPIRE].

[40] G. Bonelli, On the tensionless limit of bosonic strings, infinite symmetries and higher spins, Nucl. Phys. B 669 (2003) 159 [hep-th/0305155] [INSPIRE].

[41] I.L. Buchbinder, A. Fotopoulos, A.C. Petkou and M. Tsulaia, Constructing the cubic interaction vertex of higher spin gauge fields, Phys. Rev. D 74 (2006) 105018 [hep-th/0609082] [INSPIRE].

[42] A. Fotopoulos and M. Tsulaia, Interacting higher spins and the high energy limit of the bosonic string, Phys. Rev. D 76 (2007) 025014 [arXiv:0705.2939] [InSPIRE].

[43] A. Fotopoulos, N. Irges, A.C. Petkou and M. Tsulaia, Higher-spin gauge fields interacting with scalars: the Lagrangian cubic vertex, JHEP 10 (2007) 021 [arXiv:0708.1399] [INSPIRE].

[44] A. Fotopoulos and M. Tsulaia, Gauge invariant Lagrangians for free and interacting higher spin fields. A review of the BRST formulation, Int. J. Mod. Phys. A 24 (2009) 1 [arXiv: 0805.1346] [INSPIRE]. 
[45] A. Sagnotti and M. Taronna, String lessons for higher-spin interactions, Nucl. Phys. B 842 (2011) 299 [arXiv: 1006.5242] [INSPIRE].

[46] D. Polyakov, Interactions of massless higher spin fields from string theory, Phys. Rev. D 82 (2010) 066005 [arXiv:0910.5338] [INSPIRE].

[47] D. Polyakov, Gravitational couplings of higher spins from string theory, Int. J. Mod. Phys. A 25 (2010) 4623 [arXiv:1005.5512] [INSPIRE].

[48] E.S. Fradkin and M.A. Vasiliev, On the gravitational interaction of massless higher spin fields, Phys. Lett. B 189 (1987) 89 [inSPIRE].

[49] E.S. Fradkin and M.A. Vasiliev, Cubic interaction in extended theories of massless higher spin fields, Nucl. Phys. B 291 (1987) 141 [INSPIRE].

[50] M.A. Vasiliev, Cubic interactions of bosonic higher spin gauge fields in $A d S_{5}$, Nucl. Phys. B 616 (2001) 106 [Erratum ibid. B 652 (2003) 407] [hep-th/0106200] [INSPIRE].

[51] K.B. Alkalaev and M.A. Vasiliev, $N=1$ supersymmetric theory of higher spin gauge fields in $A d S_{5}$ at the cubic level, Nucl. Phys. B 655 (2003) 57 [hep-th/0206068] [INSPIRE].

[52] M.A. Vasiliev, Cubic vertices for symmetric higher-spin gauge fields in $(A) d S_{d}$, Nucl. Phys. B 862 (2012) 341 [arXiv:1108.5921] [INSPIRE].

[53] M.A. Vasiliev, Nonlinear equations for symmetric massless higher spin fields in (A) $d S_{d}$, Phys. Lett. B 567 (2003) 139 [hep-th/0304049] [INSPIRE].

[54] X. Bekaert, E. Joung and J. Mourad, On higher spin interactions with matter, JHEP 05 (2009) 126 [arXiv:0903.3338] [INSPIRE].

[55] X. Bekaert and E. Meunier, Higher spin interactions with scalar matter on constant curvature spacetimes: conserved current and cubic coupling generating functions, JHEP 11 (2010) 116 [arXiv:1007.4384] [INSPIRE].

[56] S.M. Kuzenko, R. Manvelyan and S. Theisen, Off-shell superconformal higher spin multiplets in four dimensions, JHEP 07 (2017) 034 [arXiv: 1701.00682] [INSPIRE].

[57] I.L. Buchbinder, S.J. Gates Jr. and K. Koutrolikos, Higher spin superfield interactions with the chiral supermultiplet: conserved supercurrents and cubic vertices, Universe 4 (2018) 6 [arXiv: 1708.06262] [INSPIRE].

[58] J. Hutomo and S.M. Kuzenko, Non-conformal higher spin supercurrents, Phys. Lett. B 778 (2018) 242 [arXiv:1710.10837] [InSPIRE].

[59] J. Hutomo and S.M. Kuzenko, The massless integer superspin multiplets revisited, JHEP 02 (2018) 137 [arXiv:1711.11364] [INSPIRE].

[60] K. Koutrolikos, P. Kočí and R. von Unge, Higher spin superfield interactions with complex linear supermultiplet: conserved supercurrents and cubic vertices, JHEP 03 (2018) 119 [arXiv: 1712.05150] [INSPIRE].

[61] J. Maldacena and A. Zhiboedov, Constraining conformal field theories with a higher spin symmetry, J. Phys. A 46 (2013) 214011 [arXiv:1112.1016] [InSPIRE].

[62] I.L. Buchbinder and S.M. Kuzenko, Ideas and methods of supersymmetry and supergravity, IOP Publishing, Bristol U.K., (1998) [INSPIRE].

[63] M. Magro, I. Sachs and S. Wolf, Superfield Noether procedure, Annals Phys. 298 (2002) 123 [hep-th/0110131] [INSPIRE]. 
[64] S.J. Gates Jr., M.T. Grisaru, M. Roček and W. Siegel, Superspace or one thousand and one lessons in supersymmetry, Front. Phys. 58 (1983) 1 [hep-th/0108200] [INSPIRE].

[65] T. Curtright, Massless field supermultiplets with arbitrary spin, Phys. Lett. B 85 (1979) 219 [INSPIRE].

[66] S.M. Kuzenko, A.G. Sibiryakov and V.V. Postnikov, Massless gauge superfields of higher half integer superspins, JETP Lett. 57 (1993) 534 [Pisma Zh. Eksp. Teor. Fiz. 57 (1993) 521] [INSPIRE].

[67] S.M. Kuzenko and A.G. Sibiryakov, Massless gauge superfields of higher integer superspins, JETP Lett. 57 (1993) 539 [Pisma Zh. Eksp. Teor. Fiz. 57 (1993) 526] [INSPIRE].

[68] S.M. Kuzenko and A.G. Sibiryakov, Free massless higher superspin superfields on the anti-de Sitter superspace, Phys. Atom. Nucl. 57 (1994) 1257 [Yad. Fiz. 57 (1994) 1326] [arXiv:1112.4612] [INSPIRE].

[69] S.J. Gates Jr. and K. Koutrolikos, On $4 D, N=1$ massless gauge superfields of arbitrary superhelicity, JHEP 06 (2014) 098 [arXiv: 1310.7385] [INSPIRE].

[70] S.J. Gates Jr. and K. Koutrolikos, On 4D, $N=1$ massless gauge superfields of higher superspin: half-odd-integer case, arXiv:1310.7386 [INSPIRE].

[71] S.J. Gates Jr. and K. Koutrolikos, On 4D, $N=1$ massless gauge superfields of arbitrary superhelicity, JHEP 06 (2014) 098 [arXiv: 1310.7385] [INSPIRE].

[72] S.J. Gates Jr. and K. Koutrolikos, From Diophantus to supergravity and massless higher spin multiplets, JHEP 11 (2017) 063 [arXiv:1707.00194] [INSPIRE].

[73] I.L. Buchbinder and K. Koutrolikos, BRST analysis of the supersymmetric higher spin field models, JHEP 12 (2015) 106 [arXiv:1510.06569] [INSPIRE]. 